\title{
Exceptional sets for self-similar fractals in Carnot groups
}

\author{
BY ZOLTÁN M. BALOGH, RETO BERGER † \\ Mathematisches Institut, Universität Bern, Sidlerstrasse 5, CH-3012 Bern, Switzerland. \\ e-mail: zoltan.balogh@math.unibe.ch, reto.berger@math.unibe.ch
}

\section{ROBERTO MONTI\$}

Dipartimento di Matematica Pura e Applicata, Università di Padova, Via Trieste, 6335121 Padova, Italy. e-mail: monti@math. unipd.it

AND JEREMY T. TYSON§

Department of Mathematics, University of Illinois at Urbana-Champaign 1409

West Green St., Urbana, IL 61801, U.S.A. e-mail: tyson@math.uiuc.edu

(Received 24 August 2009; revised 8 December 2009)

\begin{abstract}
We consider self-similar iterated function systems in the sub-Riemannian setting of Carnot groups. We estimate the Hausdorff dimension of the exceptional set of translation parameters for which the Hausdorff dimension in terms of the Carnot-Carathéodory metric is strictly less than the similarity dimension. This extends a recent result of Falconer and Miao from Euclidean space to Carnot groups.
\end{abstract}

\section{Introduction}

\subsection{Preliminaries}

One of the most common ways to construct and describe fractal sets (cf. $[\mathbf{1 1}, \mathbf{1 7}]$ ) is by the action of a system of contractions-known as an iterated function system (IFS) - on a metric space. Let $(X, d)$ be a complete metric space and $\mathbf{f}=\left\{f_{1}, \ldots, f_{M}\right\}$ be a finite set of contraction mappings on $X$. That is, for each $j=1, \ldots, M$, the map $f_{j}: X \rightarrow X$ satisfies

$$
d\left(f_{j}(x), f_{j}(y)\right) \leqslant r_{j} d(x, y)
$$

$\dagger$ Supported by Swiss National Science Foundation, European Research Council Project GALA and European Science Foundation Project HCAA.

$\ddagger$ Supported by the Mathematisches Insitut, Universität Bern.

$\S$ Supported by US National Science Foundation Grants DMS-0555869 and DMS-0901620. 
for all $x, y \in X$ and some $r_{j}<1$. Then the (unique) nonempty, compact set $K=K_{\mathrm{f}} \subset X$ which satisfies

$$
\bigcup_{j=1}^{M} f_{j}(K)=K
$$

is called the invariant set of $\mathbf{f}$; in most cases it is a fractal.

When equality holds in (1.1) for all $x, y \in X$, the mapping $f_{j}$ is called a similarity transformation of $X$ with similarity ratio $r_{j}$. In this case the underlying invariant set is selfsimilar. The Hausdorff dimensions of self-similar invariant sets can be calculated using the celebrated Moran-Hutchinson theorem $[\mathbf{1 1}, \mathbf{1 5}]$. According to this theorem, if $\mathbf{f}$ satisfies the open set condition, i.e., there exists a nonempty open set $U \subset X$ with the property that $f_{1}(U), \ldots, f_{M}(U)$ are disjoint subsets of $U$, then the Hausdorff dimension of $K$ is equal to the similarity dimension of $\mathbf{f}$ :

$$
\operatorname{dim} K=d_{\mathbf{f}} .
$$

Here the similarity dimension $d_{\mathbf{f}}$ is defined as the (unique) positive number satisfying

$$
\sum_{j=1}^{M} r_{j}^{d_{\mathrm{f}}}=1 .
$$

In its original formulation [15] this theorem was set in the Euclidean space $X=\mathbb{R}^{N}$. It continues to hold in metric spaces satisfying a doubling condition, see [2].

In the Euclidean case, similarities are precisely described by the formula

$$
f(x)=A x+a,
$$

where $A$ is a linear map in the conformal group $\mathbb{R}_{+} \cdot O(N)$ and $a \in \mathbb{R}^{N}$ is a translation vector. By results of Falconer [10], [12] and Solomyak [22], the equality of Hausdorff and similarity dimensions persists almost surely for generic IFS. More precisely, let $\mathbf{f}$ be a selfsimilar IFS with maps $f_{j}(x)=A_{j} x+a_{j}$ as in (1.3) and let $\mathbf{a}:=\left(a_{1}, \ldots, a_{M}\right) \in \mathbb{R}^{N M}$. Denote the invariant set for $\mathbf{f}$ by $K_{\mathrm{a}}$. According to [10] and [22], if $r_{j}=\left\|A_{j}\right\|<1 / 2$ for $j=1, \ldots, M$, then the equality $\operatorname{dim} K_{\mathbf{a}}=d_{\mathbf{f}}$ holds for $\mathcal{L}^{N M}$ a.e. $\mathbf{a} \in \mathbb{R}^{N M}$.

In a recent paper [8], Falconer and Miao estimated the size of the exceptional set of parameters $\mathbf{a} \in \mathbb{R}^{N M}$ for which the equality $\operatorname{dim} K_{\mathbf{a}}=d_{\mathbf{f}}$ fails. To recall their result, let us denote by

$$
E(s)=\left\{\mathbf{a} \in \mathbb{R}^{N M}: \operatorname{dim} K_{\mathbf{a}}<s\right\}
$$

the exceptional set of parameters associated to a value $s \leqslant d_{\mathbf{f}}$. Then, according to the main result of [8], the estimate

$$
\operatorname{dim} E(s) \leqslant N M-(N-s)
$$

holds for $0<s \leqslant d_{\mathbf{f}} \leqslant N$. We emphasize that the setting of $[\mathbf{8}, \mathbf{1 0}, \mathbf{1 2}$ and 22$]$ is significantly more general, encompassing arbitrary self-affine fractals: we have merely stated the special case of their results covering the self-similar situation.

The purpose of the present paper is to generalize (1.4) to the sub-Riemannian metric setting of Carnot groups. Our main theorem is Theorem 1.1. Recent years have seen a rapid development of geometric measure theory in the setting of Carnot-Carathéodory spaces, where Carnot groups play a major role. Carnot-Carathéodory spaces themselves have a fractal nature as their Hausdorff dimension is typically strictly greater than the topological 
dimension [14]. The metric in these spaces is defined by vector fields satisfying the Hörmander condition and is useful for describing various nonlinear phenomena as seen e.g. in [20]. The literature on geometric measure theory in Carnot groups and CarnotCarathéodory spaces is by now too extensive to recall in detail. We note in particular recent work of Mattila and coauthors, $[18,19]$, on tangent measures and rectifiability in the Heisenberg group. Following work of Strichartz [23], Balogh, Höfer-Isenegger, and Tyson $[1,3]$, studied fractal geometry in the Heisenberg group. Recently Balogh, Tyson and Warhurst $[4,5]$ extended the results of Falconer and Solomyak to the setting of Carnot groups. In order to state our main theorem precisely, we shall next recall the primary results from [5] and fix notation to be used throughout the paper.

\subsection{Statement of the main result}

As references for the following basic material on stratified Lie algebras and Carnot groups, we recommend Corwin-Greenleaf [7] and Folland-Stein [13]. Let $\mathrm{g}$ be a finite dimensional, stratified, nilpotent Lie algebra of step $S \geqslant 2$ and dimension $N$. We denote by $[\cdot, \cdot]$ the commutator in $\mathrm{g}$. Then there are vector spaces $\mathrm{g}_{1}, \ldots, \mathrm{g}_{S} \subset \mathrm{g}$ such that

$$
\mathrm{g}=\mathrm{g}_{1} \oplus \cdots \oplus \mathrm{g}_{S}
$$

and for all $i=1, \ldots, S$ we have

$$
\left[\mathrm{g}_{1}, \mathrm{~g}_{i}\right]= \begin{cases}\mathrm{g}_{i+1}, & \text { if } i=1, \ldots, S-1, \\ \{0\} & \text { if } i=S .\end{cases}
$$

The first layer $g_{1}$, which generates the full Lie algebra $g$, is called the horizontal layer. We denote by $m_{i} \geqslant 1$ the dimension of $\mathrm{g}_{i}, i=1, \ldots, S$, and we let $N=m_{1}+\cdots+m_{S}$. According to (1.5), any element $x \in \mathrm{g}$ has the unique decomposition

$$
x=x^{(1)}+\cdots+x^{(S)},
$$

with $x^{(i)} \in \mathrm{g}_{i}, i=1, \ldots, S$. For any $r>0$, the linear mapping $\delta_{r}: \mathrm{g} \rightarrow \mathrm{g}$

$$
\delta_{r}(x)=r x^{(1)}+\cdots+r^{S} x^{(S)}
$$

is a Lie algebra automorphism, which is called dilation by $r>0$.

The Baker-Campbell-Hausdorff formula provides a group operation $*: \mathrm{g} \times \mathrm{g} \rightarrow \mathrm{g}$ which makes $\mathbb{G}=(\mathrm{g}, *)$ a nilpotent and stratified Lie group of step $S$. Such groups are called Carnot groups. More details can be found in Section 3.

We fix on $\mathbb{G}$ a distance function $d$ with the following properties:

(i) $d(z * x, z * y)=d(x, y)$ for all $x, y, z \in \mathbb{G}$ (i.e. $d$ is left invariant);

(ii) $d\left(\delta_{r}(x), \delta_{r}(y)\right)=r d(x, y)$ for all $x, y \in \mathbb{G}$ and $r>0$ (i.e. $d$ is 1-homogeneous with respect to the dilations $\delta_{r}$ ).

Any such metric we will call a Carnot-Carathéodory or sub-Riemannian metric on $\mathbb{G}$. The existence of such metrics is well known. For instance, one may use the metric constructed by taking the infimum of lengths of horizontal curves, see [14] or [6]. (Recall that a piecewise $C^{1}$ curve taking values in $\mathbb{G}$ is called horizontal if its tangent vectors lie in the subbundle $H \mathbb{G}$ of $T \mathbb{G}$ spanned by the horizontal layer $\mathrm{g}_{1}$, viewed as a space of left invariant vector fields. Length of such a curve is computed with respect to a fixed smoothly varying family of inner products defined on the subbundle $H \mathbb{G}$.) Other more explicit sub-Riemannian metrics can be constructed directly, such as the Korányi metric on the Heisenberg group $\mathbb{H}^{1}$ (see 
Example 6.1). For our purposes the precise choice of metric is not important since all subRiemannian metrics on a given Carnot group are bi-Lipschitz equivalent and the results of this paper are bi-Lipschitz invariant. Any sub-Riemannian metric $d$ on $\mathbb{G}$ is complete.

The topological dimension of $(\mathbb{G}, d)$ is equal to $N$ (the dimension of the underlying Lie algebra). On the other hand, the Hausdorff dimension of $(\mathbb{G}, d)$ is equal to

$$
Q=m_{1}+2 m_{2}+\cdots+S m_{S},
$$

the homogeneous dimension of $\mathbb{G}$. We observe a discrepancy between these two dimensions: $Q>N$. This discrepancy persists for arbitrary subsets $A \subset \mathbb{G}$. A quantitative description of this dimension discrepancy was presented in [5] in terms of certain piecewise linear dimension comparison functions $\beta_{ \pm}:[0, N] \rightarrow[0, Q]$. More precisely, it was shown [5, theorem 2.4] that

$$
\beta_{-}\left(\operatorname{dim}_{E} A\right) \leqslant \operatorname{dim}_{c c} A \leqslant \beta_{+}\left(\operatorname{dim}_{E} A\right)
$$

for every $A \subset \mathbb{G}$. Here we denote by $\operatorname{dim}_{c c}$ the Hausdorff dimension in the metric space $(\mathbb{G}, d)$, i.e. with respect to the Carnot-Carathéodory metric, and by $\operatorname{dim}_{E}$ the Hausdorff dimension in the usual Euclidean metric on the underlying space $\mathbb{R}^{N}$.

Furthermore [5, theorem 2.6], the estimates in (1.10) are sharp as shown by explicit examples constructed using IFS on $\mathbb{G}$. This observation is closely related with dimension formulae for generic IFS generalizing the above Falconer-Solomyak theorem. The existence of a self-similar dilation structure $\left(\delta_{r}\right)_{r>0}$ on Carnot groups which interacts well with the group law $*$ and the left invariant metric $d$ makes such spaces natural settings in which to study self-similar iterated function systems.

To be more precise, consider a system of similarities $\mathbf{f}=\left\{f_{1}, \ldots, f_{M}\right\}$ in $\mathbb{G}$ of the form

$$
f_{j}(x)=a_{j} * \delta_{r_{j}}(x),
$$

$j=1, \ldots, M$, where $\mathbf{a}=\left(a_{1}, \ldots, a_{M}\right) \in \mathbb{G}^{M}$ is an $M$-tuple of translation vectors and $\mathbf{r}=$ $\left(r_{1}, \ldots, r_{M}\right) \in(0,1)^{M}$ is an $M$-tuple of contraction ratios. The invariant set $K_{\mathbf{f}}$ depends on $\mathbf{a}$ and $\mathbf{r}$. Fixing the contraction ratios, we denote this self-similar set by $K_{\mathbf{a}}$ with no reference to $\mathbf{r}$. We associate to $K_{\mathbf{a}}$ the Carnot-Carathéodory similarity dimension $d_{\mathbf{r}}$ satisfying $(1 \cdot 2)$. Then (see [5, theorem 2.8]) the following dimension formulas are valid for almost every $\mathbf{a} \in \mathbb{G}^{M}:$ if $d_{\mathbf{r}} \leqslant Q$ and $r_{j}<1 / 2$ for all $j=1, \ldots, M$, then:

(a) $\operatorname{dim}_{c c} K_{\mathbf{a}} \leqslant d_{\mathbf{r}}$ for all $\mathbf{a} \in \mathbb{G}^{M}$;

(b) $\operatorname{dim}_{E} K_{\mathbf{a}} \leqslant \beta_{-}^{-1}\left(d_{\mathbf{r}}\right)$ for all $\mathbf{a} \in \mathbb{G}^{M}$;

(c) $\operatorname{dim}_{c c} K_{\mathbf{a}}=d_{\mathbf{r}}$ for a.e. $\mathbf{a} \in \mathbb{G}^{M}$;

(d) $\operatorname{dim}_{E} K_{\mathbf{a}}=\beta_{-}^{-1}\left(d_{\mathbf{r}}\right)$ for a.e. $\mathbf{a} \in \mathbb{G}^{M}$.

The measure on $\mathbb{G}^{M}$ in question is the $M$-fold product Haar measure, which is equivalent with either the Lebesgue measure $\mathcal{L}^{N M}$ on $\mathbb{G}^{M}=\mathbb{R}^{N M}$ or to the Hausdorff measure $\mathcal{H}^{Q M}$ in the product Carnot-Carathéodory metric.

The most interesting statements above are (c) and (d) which give almost sure dimension formulas in terms of the Carnot-Carathéodory and also in terms of the Euclidean metric. In particular, $K_{\mathbf{a}}$ is almost surely a horizontal set (in the terminology introduced in [5]), i.e., $\operatorname{dim}_{c c} K_{\mathbf{a}}=\beta_{-}\left(\operatorname{dim}_{E} K_{\mathbf{a}}\right)$.

We are interested in quantifying the previous statement by measuring precisely (in terms of Hausdorff measures on the parameter space) the set of values a for which $K_{\mathbf{a}}$ fails to be horizontal. See Problem 6.2. In this paper, we take a first step in this direction by proving 
a result analogous to the Falconer-Miao estimate (1.4) on the size of the exceptional set of parameters $\mathbf{a} \in \mathbb{G}^{M}$ for which estimate (c) above fails. To do so, we introduce for $s \leqslant d_{\mathbf{r}} \leqslant$ $Q$ the exceptional set

$$
E(s)=\left\{\mathbf{a} \in \mathbb{G}^{M}: \operatorname{dim}_{c c} K_{\mathbf{a}}<s\right\} .
$$

According to the preceding discussion, the $(Q M)$-dimensional Carnot-Carathéodory Hausdorff measure (equivalently, the $(N M)$-dimensional Euclidean Hausdorff measure) of $E(s)$ vanishes for all $s \leqslant d_{\mathbf{r}}$. The primary result of this paper is the following theorem.

THEOREM 1.1. Let $\mathbb{G}$ be a Carnot group of topological dimension $N$ and homogeneous dimension $Q$. Fix $M$ positive real numbers $0<r_{j}<1 / 2, j=1, \ldots, M$, and let $d_{\mathbf{r}}$ be the corresponding similarity dimension. Then

$$
\operatorname{dim}_{c c} E(s) \leqslant Q M-\left(N-\beta_{-}^{-1}(s)\right)=Q M-\beta_{+}^{-1}(Q-s)
$$

for $s<d_{\mathbf{r}} \leqslant Q$.

COROLlaRY 1.1. We have $\operatorname{dim}_{c c} E\left(d_{\mathbf{r}}\right) \leqslant Q M-\beta_{+}^{-1}\left(Q-d_{\mathbf{r}}\right)$.

Since in the Euclidean case $Q=N$ and $\beta_{-}=\beta_{+}=\left.\mathrm{id}\right|_{[0, N]},(1 \cdot 12)$ extends the FalconerMiao estimate (1.4). For the second equality in $(1 \cdot 12)$ we observe the identity

$$
Q-\beta_{+}(\alpha)=\beta_{-}(N-\alpha), \quad 0 \leqslant \alpha \leqslant N,
$$

which follows from the definitions of $\beta_{ \pm}$(see $(5 \cdot 1)$ and $\left.(5 \cdot 3)\right)$. Note that $\beta_{-}:[0, N] \rightarrow$ $[0, Q]$ is an increasing homeomorphism, thus $\beta_{-}^{-1}(s)<N$ whenever $s<Q$. Corollary $1 \cdot 1$ improves on the result from [5] by showing that the dimension of the exceptional set $E\left(d_{\mathbf{r}}\right)$ is bounded away from $Q M$ whenever $d_{\mathbf{r}}<Q$. Theorem 1.1 may be useful in solving the more difficult Problem $6 \cdot 2$ on exceptional sets; see the discussion following the statement of that problem for more information. In Example $6 \cdot 1$ we illustrate our theorem by indicating the precise exceptional sets for the almost sure dimension results (b) and (d) above, in the case of two-element IFS's on the first Heisenberg group $\mathbb{H}^{1}$.

As additional motivation for the study of the exceptional sets problem in this nonlinear environment, we observe that self-similar IFS in Carnot groups of high step are comprised of nonlinear, nonconformal maps of the underlying Euclidean space $\mathbb{R}^{N}$. Indeed, the appearance of the group operation in (1.11) means that the constituent maps $f_{i}$ of the IFS $\mathbf{f}$, when viewed as maps of $\mathbb{R}^{N}$, are polynomial maps of degree at most (and typically equal to) $S-1$. Our results from [5] and in the present paper thus provide almost sure statements and estimates on exceptional sets for parameterized families of nonlinear, nonconformal IFS in Euclidean space. We point out that other approaches to the exceptional sets problem exist in the literature which apply in nonlinear settings; see for example [21] for the use of transversality in this context.

To conclude this introduction we briefly explain the idea of the proof of Theorem $1 \cdot 1$. We note first that the points in $K_{\mathbf{a}}$ can be parameterized by words as follows. Denote by $W_{\infty, M}$ the set of all words $\omega=\omega_{1} \omega_{2} \omega_{3} \cdots$ with countably many letters $\omega_{n}$, each selected from the alphabet $\{1, \ldots, M\}$, and define the projection map $\pi_{\mathbf{a}}$ from $W_{\infty, M}$ to $K_{\mathbf{a}}$ by

$$
\pi_{\mathbf{a}}(\omega)=\lim _{\ell \rightarrow \infty} f_{\omega_{1}} \circ \cdots \circ f_{\omega_{\ell}}\left(x_{0}\right),
$$

where $x_{0}$ is any point in $\mathbb{G}$. The mapping $\pi_{\mathbf{a}}$ does not depend on $x_{0}$. Our approach follows the one of Falconer and Miao [8] and goes back to the idea of Falconer [10]. The main 
point is to realize a transition from the parameter space onto the dynamical space. More precisely, for any pair of words $\omega, v \in W_{\infty, M}$ such that $\omega_{1}=1$ the transition map $T_{\omega, v}=$ $\left(T_{\omega, v}^{1}, \ldots, T_{\omega, v}^{M}\right): \mathbb{G}^{M} \rightarrow \mathbb{G}^{M}$ is defined by

$$
T_{\omega, v}^{j}(\mathbf{a})= \begin{cases}\pi_{\mathbf{a}}(\omega)^{-1} * \pi_{\mathbf{a}}(v) & \text { for } j=1, \\ a_{j} & \text { for } j \neq 1,\end{cases}
$$

for all $j=1, \ldots, M$.

In the Euclidean case [8], the transition map is an invertible linear map with norm bounded from below by a positive constant independent of the words $\omega$ and $v$, as soon as $\omega_{1} \neq v_{1}$. In our case the transition map is nonlinear and is not bi-Lipschitz continuous with respect to the product metric on $\mathbb{G}^{M}$ that is generated by the Carnot-Carathéodory metric of $\mathbb{G}$. The main technical difficulty of our work is to deal with the complex nonlinearity of the mapping. The solution of the problem is that we will show that $T_{\omega, v}$ is bi-Lipschitz continuous on $\mathbb{G}^{M}$ with respect to the Euclidean metric. Passing from the Euclidean metric to the CarnotCarathéodory metric is done by a covering argument akin to the technique used in [5]. The discrepancy between the two metrics resurfaces in the appearance of the dimension comparison functions $\beta_{ \pm}$in the statement of Theorem 1.1.

The paper is organized as follows. In the second section we present results on symbolic dynamics and of potential theoretic nature which will be used in the proof. In section three we develop an iterated version of the Baker-Campbell-Hausdorff formula in order to deal with the infinite products appearing in the formula for the transition map as seen in (1.13) and (1.14). The results obtained in this section may be of independent interest in the theory of Carnot groups. In the fourth section we prove the main properties of the transition map culminating in its Euclidean bi-Lipschitz continuity. In section five we prove Theorem 1.1. The last section is for comments and open questions.

\section{Symbolic dynamics, energy and Hausdorff dimension}

\subsection{Symbolic dynamics}

We review the language and notation of symbolic dynamics for iterated function systems. The material in this section is mostly standard. A useful reference is Kigami [16].

We may parameterize the points of the invariant set of an iterated function system in a natural fashion by taking into account its dynamical construction. To this end we define abstract word spaces. For $\ell, n \in \mathbb{N}$, we define the following three sets:

$$
\begin{aligned}
& W_{\ell, n}=\{u \mid u:\{1,2, \ldots, \ell\} \rightarrow\{1,2, \ldots, n\}\}, \\
& W_{*, n}=\bigcup_{\ell \in \mathbb{N}} W_{\ell, n} \cup\{e: \varnothing \rightarrow\{1,2, \ldots, n\}\}
\end{aligned}
$$

and

$$
W_{\infty, n}=\{w \mid w: \mathbb{N} \rightarrow\{1,2, \ldots, n\} .
$$

The notation has a natural generalization to the case $n=\infty$.

An element $u \in W_{\ell, n}$ is called a finite word of length $\ell(u)=\ell$, where $e$ is the so called empty word of length zero. Elements of $W_{\infty, n}$ are called infinite words. The set $W_{*, n}$ is the collection of all finite words. The $k$ th letter $w_{k}$ of a word $w$ is simply the value $w(k)$. We will write

$$
u=u_{1} u_{2} \cdots u_{\ell}
$$


for $u \in W_{\ell, n}$ and

$$
w=w_{1} w_{2} w_{3} \ldots
$$

for $w \in W_{\infty, n}$. Concatenation of a finite word with a finite or infinite word is defined in the obvious way: if $u=u_{1} u_{2} \cdots u_{\ell} \in W_{*, n}$ and $w=w_{1} w_{2} \cdots \in W_{*, n} \cup W_{\infty, n}$, then

$$
u w=u_{1} u_{2} \cdots u_{\ell} w_{1} w_{2} w_{3} \cdots .
$$

The longest common prefix $v \wedge w$ of two words $v, w \in W_{*, n} \cup W_{\infty, n}$ is defined by

$$
v \wedge w=\left\{\begin{array}{lll}
e & \text { if } & v_{1} \neq w_{1} \\
u & \text { if } & v=u v^{\prime} \text { and } w=u w^{\prime} \text { and } v_{1}^{\prime} \neq w_{1}^{\prime} \\
v & \text { if } \quad v=w .
\end{array}\right.
$$

The shift operator $\sigma: W_{\infty, n} \rightarrow W_{\infty, n}$ and its $n$ right inverses $\sigma_{j}: W_{\infty, n} \rightarrow W_{\infty, n}$, are given by the formulas $\sigma\left(w_{1} w_{2} w_{3} \cdots\right)=w_{2} w_{3} w_{4} \cdots$ and $\sigma_{j}\left(w_{1} w_{2} w_{3} \cdots\right)=j w_{1} w_{2} \cdots$. For $u=u_{1} \cdots u_{\ell} \in W_{*, n}$ we set $\sigma_{u}:=\sigma_{u_{1}} \circ \cdots \circ \sigma_{u_{\ell}}$.

The space $W_{\infty, n}$ of infinite words is naturally topologized with the product topology coming from the discrete topology on $\{1,2, \ldots, n\}$. This topology is metrizable, an explicit generating metric being

$$
d_{\infty}(v, w):= \begin{cases}2^{-\ell(v \wedge w)} & \text { if } v \neq w \\ 0 & \text { if } v=w .\end{cases}
$$

By the Tychonoff Product Theorem, $\left(W_{\infty, n}, d_{\infty}\right)$ is compact.

To each word $u \in W_{*, n}$ we associate the cylinder set

$$
\Omega_{u}:=\left\{u w \in W_{\infty, n} \mid w \in W_{\infty, n}\right\} .
$$

We observe that every open ball in $\left(W_{\infty, n}, d_{\infty}\right)$ coincides with a cylinder set $\Omega_{u}$ for some $u \in W_{*, n}$.

Let $\alpha: W_{*, n} \rightarrow[0,+\infty)$ satisfy the consistency relation

$$
\alpha(u)=\sum_{j=1}^{n} \alpha(u j)
$$

for all $u \in W_{*, n}$. Then there exists a unique Borel measure $\lambda: \mathcal{P}\left(W_{\infty, n}\right) \rightarrow[0,+\infty]$ whose value on cylinder sets is given by $\lambda\left(\Omega_{u}\right)=\alpha(u)$ for all $u \in W_{*, n}$. In particular,

$$
\lambda\left(W_{\infty, n}\right)=\alpha(e) .
$$

Let $\mathbf{f}=\left\{f_{1}, \ldots, f_{M}\right\}$ be an iterated function system defined on a complete metric space $(X, d)$. For each $j, 1 \leqslant j \leqslant M$, let $r_{j}$ be the contraction ratio associated to $f_{j}$. For $u=$ $u_{1} u_{2} \cdots u_{\ell} \in W_{*, M}$ we define a map $f_{u}: X \rightarrow X$ and a real number $r_{u}$ by

$$
f_{u}=f_{u_{1}} \circ f_{u_{2}} \circ \cdots \circ f_{u_{\ell}} \text { and } r_{u}=r_{u_{1}} r_{u_{2}} \cdots r_{u_{\ell}} \text {. }
$$

We denote the invariant set for $\mathbf{f}$ by $K_{\mathbf{f}}$. We equip $W_{\infty, M}$ with the product topology as described in the previous section.

The map $\pi_{\mathbf{f}}: W_{\infty, M} \rightarrow K_{\mathbf{f}}$ given by

$$
\pi_{\mathbf{f}}(w):=\lim _{\ell \rightarrow \infty} f_{w_{1} w_{2} \cdots w_{\ell}}\left(x_{0}\right)
$$


where $x_{0}$ is an arbitrary point in $X$, is continuous and surjective. It is easy to check that $\pi_{\mathbf{f}}$ is independent of $x_{0}$. We observe the key relation

$$
\pi_{\mathbf{f}} \circ \sigma_{u}=f_{u} \circ \pi_{\mathbf{f}}, \quad u \in W_{*, n} .
$$

The similarity dimension for the iterated function system $\mathbf{f}$ is the unique nonnegative real number $d_{\mathbf{f}}$ for which

$$
\sum_{j=1}^{M} r_{j}^{d_{\mathbf{f}}}=1
$$

As this value depends only on the vector $\mathbf{r}=\left(r_{1}, \ldots, r_{M}\right)$ of contraction ratios associated to $\mathbf{f}$, we will write $d_{\mathbf{r}}$ for the similarity dimension of $\mathbf{f}$. Define $\alpha: W_{*, M} \rightarrow[0,+\infty)$ by the formula $\alpha(u):=r_{u}^{d_{\mathrm{r}}}$. This function satisfies $(2 \cdot 1)$ since

$$
\sum_{j=1}^{M} \alpha(u j)=\sum_{j=1}^{M} r_{u j}^{d_{\mathbf{r}}}=r_{u}^{d_{\mathbf{r}}} \sum_{j=1}^{M} r_{j}^{d_{\mathrm{r}}}=r_{u}^{d_{\mathrm{r}}}=\alpha(u) .
$$

Hence there exists a Borel measure $\lambda_{\mathbf{r}}$ on $W_{\infty, M}$ with

$$
\lambda_{\mathbf{r}}\left(\Omega_{u}\right)=r_{u}^{d_{\mathbf{r}}}
$$

for all $u \in W_{*, M}$. By $(2 \cdot 2), \lambda_{\mathbf{r}}$ is a probability measure.

\subsection{Energy and dimension}

Let $(X, d)$ be a complete metric space and $s$ a positive real number. The s-energy of a mass distribution $v$ supported on a compact set $K \subset X$ is defined to be

$$
\mathcal{E}_{s}(K, v):=\int_{K} \int_{K} d(x, y)^{-s} d \nu(x) d v(y) .
$$

The following theorem relates energy to Hausdorff dimension. For a proof, see [9].

Proposition $2 \cdot 1$ (s-energy versus Hausdorff dimension). Let $K \subset X$ be a Borel set and let $s$ be a positive real number. Then the following holds:

(a) if the s-energy $\mathcal{E}_{s}(K, v)$ is finite for some mass distribution $v$ supported on $K$, then $\operatorname{dim}_{c c} K \geqslant s$

(b) if $\mathcal{H}^{s}(K)>0$, then for each $t, 0<t<s$, there exists a mass distribution $v$ supported on $K$ whose t-energy $\mathcal{E}_{t}(K, v)$ is finite.

Let $\mathbb{G}$ be a Carnot group. Denote by $\tau_{a}: \mathbb{G} \rightarrow \mathbb{G}$ the operation of left translation by $a \in \mathbb{G}$, i.e., $\tau_{a}(p)=a * p$. Fix positive real numbers $r_{j}<1, j=1, \ldots, M$, and let $r_{\text {max }}<1$ denote the largest of these values. We denote by $d_{\mathbf{r}}$ the similarity dimension for the $M$ tuple $\mathbf{r}=\left(r_{1}, \ldots, r_{M}\right)$. We will always assume that $M \geqslant 2$ which implies that $d_{\mathbf{r}}>0$. For $\mathbf{a}=\left(a_{1}, \ldots, a_{M}\right) \in \mathbb{G}^{M}$ we denote the self-similar set for the iterated function system $\left\{\tau_{a_{1}} \circ \delta_{r_{1}}, \ldots, \tau_{a_{M}} \circ \delta_{r_{M}}\right\}$ by $K_{\mathbf{a}}$. Let $\pi_{\mathbf{a}}$ be the symbolic representation map from $W_{\infty, M}$ to $K_{\mathrm{a}}$ as in (2.3).

PROPOSITION 2.2 (Finiteness of the $s$-energy). Let $s<d_{\mathbf{r}}$ be a positive real number. If there exists a measure $\mu$ on $\mathbb{G}^{M}$ such that the inequality

$$
\int_{B_{R}^{M}} d\left(\pi_{\mathbf{a}}(v), \pi_{\mathbf{a}}(w)\right)^{-s} d \mu(\mathbf{a}) \leqslant L r_{v \wedge w}^{-s}
$$


holds for all $R>0$ and all distinct words $v, w \in W_{\infty, M}$ with constant $L=L(\mathbb{G}, R)$ independent of $v$ and $w$, then $\operatorname{dim}_{c c} K_{\mathbf{a}} \geqslant s$ for $\mu$-almost every $\mathbf{a} \in \mathbb{G}^{M}$.

Proof. Since $d_{\mathbf{r}}$ is positive, $\sum_{j=1}^{M} r_{j}^{2 d_{\mathbf{r}}}<1$. We consider the Borel measure $\lambda_{\mathbf{r}}$ on $W_{\infty, M}$ described in subsection 2.1. The product measure $\lambda_{\mathbf{r}} \otimes \lambda_{\mathbf{r}}$ on the diagonal $\Delta:=\{(v, w) \in$ $\left.W_{\infty, M}^{2} \mid v=w\right\}$ vanishes, because $\Delta \subset \bigcup_{u \in W_{\ell, M}} \Omega_{u} \times \Omega_{u}$ for each $\ell$ and therefore

$$
\left(\lambda_{\mathbf{r}} \otimes \lambda_{\mathbf{r}}\right)(\Delta) \leqslant \sum_{u \in W_{\ell, M}} \lambda_{\mathbf{r}}\left(\Omega_{u}\right)^{2}=\sum_{u \in W_{\ell, M}} r_{u}^{2 d_{\mathbf{r}}}=\left(\sum_{j=1}^{M} r_{j}^{2 d_{\mathbf{r}}}\right)^{\ell} \stackrel{\ell \rightarrow \infty}{\longrightarrow} 0 .
$$

Using this fact and (2.6) we get

$$
\begin{aligned}
& \int_{W_{\infty, M}^{2}} \int_{B_{R}^{M}} d\left(\pi_{\mathbf{a}}(v), \pi_{\mathbf{a}}(w)\right)^{-s} d \mu(\mathbf{a}) d\left(\lambda_{\mathbf{r}} \otimes \lambda_{\mathbf{r}}\right)(v, w) \\
& \quad \leqslant L(\mathbb{G}, R) \int_{W_{\infty, M}^{2} \backslash \Delta} r_{v \wedge w}^{-s} d\left(\lambda_{\mathbf{r}} \otimes \lambda_{\mathbf{r}}\right)(v, w) .
\end{aligned}
$$

The integrand $\varphi(v, w)=r_{v \wedge w}^{-s}$ in the latter integral has the property that

$$
\varphi\left(W_{\infty, M}^{2} \backslash \Delta\right)=\left\{r_{u}^{-s} \mid u \in W_{*, M}\right\}
$$

is a countable subset of $\mathbb{R}$, since $W_{*, M}$ is countable. Thus

$$
\begin{aligned}
& \int_{W_{\infty, M}^{2} \backslash \Delta} r_{v \wedge w}^{-s} d\left(\lambda_{\mathbf{r}} \otimes \lambda_{\mathbf{r}}\right)(v, w)=\sum_{u \in W_{*, M}} r_{u}^{-s}\left(\lambda_{\mathbf{r}} \otimes \lambda_{\mathbf{r}}\right)\left(\left\{(v, w) \in W_{\infty, M}^{2} \backslash \Delta \mid v \wedge w=u\right\}\right) \\
& \leqslant \sum_{u \in W_{s, M}} r_{u}^{-s}\left(\lambda_{\mathbf{r}} \otimes \lambda_{\mathbf{r}}\right)\left(\Omega_{u}^{2}\right)=\sum_{\ell \in \mathbb{N}} \sum_{u \in W_{\ell, M}} r_{u}^{d_{\mathbf{r}}-s} \lambda_{\mathbf{r}}\left(\Omega_{u}\right) \\
& \leqslant \sum_{\ell \in \mathbb{N}} r_{\max }{ }^{\left(d_{\mathbf{r}}-s\right) \ell} \underbrace{\sum_{u \in W_{\ell, M}} \lambda_{\mathbf{r}}\left(\Omega_{u}\right)}_{=\lambda_{\mathbf{r}}\left(W_{\infty, M}\right)=1}=\sum_{\ell \in \mathbb{N}} r_{\max }{ }^{\left(d_{\mathbf{r}}-s\right) \ell}
\end{aligned}
$$

which is finite since $s<d_{\mathbf{r}}$ and $r_{\max }<1$. Applying Fubini's theorem, we see that

$$
\int_{B_{R}^{M}} \int_{W_{\infty, M}} \int_{W_{\infty, M}} d\left(\pi_{\mathbf{a}}(v), \pi_{\mathbf{a}}(w)\right)^{-s} d \lambda_{\mathbf{r}}(v) d \lambda_{\mathbf{r}}(w) d \mu(\mathbf{a})
$$

is finite. The image measure $v_{\mathbf{a}}=\left(\pi_{\mathbf{a}}\right)_{\#}\left(\lambda_{\mathbf{r}}\right)$ is a mass distribution supported on $K_{\mathbf{a}}$, since $0<\lambda_{\mathbf{r}}\left(W_{\infty, M}\right)=1<+\infty$. From (2.8) it follows that the $s$-energy

$$
\int_{K_{\mathbf{a}}} \int_{K_{\mathbf{a}}} d(x, y)^{-s} d \nu_{\mathbf{a}}(x) d \nu_{\mathbf{a}}(y)=\int_{W_{\infty, M}} \int_{W_{\infty, M}} d\left(\pi_{\mathbf{a}}(v), \pi_{\mathbf{a}}(w)\right)^{-s} d \lambda_{\mathbf{r}}(v) d \lambda_{\mathbf{r}}(w)
$$

is finite for $\mu$-almost every $a \in B_{R}^{M}$. The result now follows from Proposition 2.1, after passing to the limit as $R \rightarrow \infty$.

\section{Iterated Baker-Campbell-Hausdorff formula}

Let $\mathrm{g}$ be a finite dimensional, stratified, nilpotent Lie algebra of step $S \geqslant 2$. For any word $w \in W_{s, n}, s, n \in \mathbb{N}$, we define the nested commutator or nested bracket of $s$ elements chosen from $x_{1}, \ldots, x_{n} \in \mathrm{g}$ and specified by $w$ in the following way

$$
\left(x_{1}, \ldots, x_{n}\right)_{w}=\left[x_{w_{1}},\left[\ldots\left[x_{w_{s-1}}, x_{w_{s}}\right] \ldots\right]\right] .
$$

The number $s \in \mathbb{N}$ is the length of the commutator. 
LEMMA 3.1. Any bracket of s elements chosen from $x_{1}, \ldots, x_{n} \in \mathrm{g}$ is a linear combination with coefficients \pm 1 of nested commutators of the form $\left(x_{1}, \ldots, x_{n}\right)_{w}$ with $w \in W_{s, n}$.

Proof. The easy proof of this lemma relies upon the Jacobi identity (see e.g. [6, p. 12]). The proof is by induction on the length of the commutator and the induction basis is the identity $\left[\left[x_{1}, x_{2}\right],\left[x_{3}, x_{4}\right]\right]=\left[x_{4},\left[x_{3},\left[x_{1}, x_{2}\right]\right]\right]-\left[x_{3},\left[x_{2},\left[x_{1}, x_{2}\right]\right]\right]$.

The Baker-Campbell-Hausdorff formula provides a Lie group structure on the Lie algebra g. From now on, the number $S \geqslant 2$ is fixed and we let

$$
V_{n}=W_{1, n} \cup \ldots \cup W_{S, n}, \quad n \in \mathbb{N},
$$

and

$$
V_{*}=\bigcup_{n \in \mathbb{N}} V_{n}
$$

THEOREM 3.2 (Baker-Campbell-Hausdorff formula). There exist constants $c_{w} \in \mathbb{Q}$, $w \in V_{2}$, making the operation $*: \mathrm{g} \times \mathrm{g} \rightarrow \mathrm{g}$ given by

$$
x_{1} * x_{2}=\sum_{w \in V_{2}} c_{w}\left(x_{1}, x_{2}\right)_{w}
$$

into an associative group law on $\mathrm{g}$.

For a proof of this theorem, see e.g. [7, theorem 1·2.1].

Example 3.1. When the step $S$ is equal to 2, formula (3.2) reads

$$
x_{1} * x_{2}=x_{1}+x_{2}+\frac{1}{2}\left[x_{1}, x_{2}\right] .
$$

When the step $S$ is equal to 3 , the formula reads

$$
x_{1} * x_{2}=x_{1}+x_{2}+\frac{1}{2}\left[x_{1}, x_{2}\right]+\frac{1}{12}\left[x_{1},\left[x_{1}, x_{2}\right]\right]-\frac{1}{12}\left[x_{2},\left[x_{1}, x_{2}\right]\right],
$$

i.e. $c_{1}=c_{2}=1, c_{12}=1 / 2, c_{21}=0, c_{112}=1 / 12, c_{212}=-1 / 12$, whereas all other constants vanish.

From now on, the constants $c_{w}$ for $w \in V_{2}$ are given by Theorem 3.2. We are not interested in their precise value. We are rather interested in the following iteration of the formula.

THEOREM 3.2 (Iterated Baker-Campbell-Hausdorff formula). For any $S \geqslant 2$ there exist a finite set $A \subset \mathbb{Q}$ and constants $c_{w} \in A, w \in V_{*}$, such that for any stratified nilpotent Lie algebra g of step $S$ we have

$$
\left(\cdots\left(\left(x_{1} * x_{2}\right) * x_{3}\right) \cdots\right) * x_{n}=\sum_{w \in V_{n}} c_{w}\left(x_{1}, \ldots, x_{n}\right)_{w}
$$

for all $x_{1}, \ldots, x_{n} \in \mathrm{g}, n \geqslant 2$.

The parentheses on the left-hand side of (3.5) can be dropped since $*$ is an associative operation. We shall denote the iterated product on the left hand side by $\underset{k=1}{\stackrel{n}{*}} x_{k}=x_{1} * \cdots * x_{n}$. 
Example 3.2. When $S=2$, formula (3.5) reads

$$
x_{1} * \cdots * x_{n}=\sum_{i=1}^{n} x_{i}+\frac{1}{2} \sum_{1 \leqslant i<j \leqslant n}\left[x_{i}, x_{j}\right]
$$

When $S=3$, the formula reads

$$
\begin{aligned}
x_{1} * \cdots * x_{n}=\sum_{i=1}^{n} x_{i} & +\frac{1}{2} \sum_{1 \leqslant i<j \leqslant n}\left[x_{i}, x_{j}\right]+\frac{1}{4} \sum_{1 \leqslant i<j<k \leqslant n}\left[x_{i},\left[x_{j}, x_{k}\right]\right] \\
& +\frac{1}{12} \sum_{i=1}^{n-1} \sum_{i<j \leqslant n}\left[x_{i},\left[x_{i}, x_{j}\right]\right]-\frac{1}{12} \sum_{j=1}^{n} \sum_{i, k>j}\left[x_{i},\left[x_{j}, x_{k}\right]\right] .
\end{aligned}
$$

This formula can be proved by induction on $n \geqslant 2$ starting from (3.4).

Proof of Theorem 3.2. We define recursively subsets $A_{n} \subset \mathbb{Q}, n \in \mathbb{N}$, in the following way. Let $A_{1}=\{0,1\}$, fix the constants $c_{w}, w \in V_{2}$, as in Theorem 3.2, and let

$$
A_{2}=\left\{c_{w} \in \mathbb{Q}: w \in V_{2}\right\}
$$

Now assume that $A_{1} \subset A_{2} \subset \cdots \subset A_{n}$ are already defined. Let $\ell_{n+1}=(n S)^{S}$ and define

$$
A_{n+1}=\left\{\sum_{h=1}^{\ell_{n+1}} d_{h} d_{0, h} d_{1, h} \cdots d_{i, h} \mid d_{h} \in\{ \pm 1\}, d_{0, h} \in A_{2}, d_{1, h}, \ldots, d_{i, h} \in A_{n}, i \leqslant S-1\right\} .
$$

For any $n \in \mathbb{N}$ and $m=1, \ldots, n$, we denote by $V_{n}^{m}$ the set of all words $w \in V_{n}$, with $w=w_{1} \cdots w_{i}$ for some $i=1, \ldots, S$, such that the set

$$
\left\{w_{1}, \ldots, w_{i}\right\} \subset\{1, \ldots, n\}
$$

contains exactly $m$ distinct elements.

We claim there is a choice of constants $c_{w} \in \mathbb{Q}$ for all $w \in V_{*}$ such that (3.5) holds for any $x_{1}, \ldots, x_{n} \in \mathrm{g}$ and moreover that

$$
c_{w} \in A_{m} \quad \text { for all } w \in V_{n}^{m}, 2 \leqslant m \leqslant \min \{n, S\} .
$$

The claim implies Theorem 3.2. Indeed, suppose that the claim holds. Then the set $A=A_{S}$ is finite and by (3.9) the formula (3.5) holds with constants $c_{w} \in A$. In fact, commutators of more than $S$ elements vanish by (1.6), and thus the sum in (3.5) ranges only over those $w \in W_{n}^{m}$ with $m \leqslant S$.

The proof of the claim is by induction on $n$. The induction basis is $n=2$. In this case, (3.5) reduces to $(3 \cdot 2)$. Now assume that (3.5) holds for $n$, i.e. there are constants $c_{w} \in A_{n}$ such that the formula holds for any $n$ elements $x_{1}, \ldots, x_{n} \in$ g. Moreover, assume that (3.9) holds. Let $x_{1}, \ldots, x_{n+1} \in \mathrm{g}$ and set $x=x_{1} * \cdots * x_{n}$. Here and in the sequel, we use the notation $x_{w}=\left(x_{1}, \ldots, x_{n}\right)_{w}$. By the inductive hypothesis,

$$
x=\sum_{w \in V_{n}} c_{w} x_{w}
$$

with $c_{w} \in A_{n}$. By (3.2) and by the associativity of the operation $*$, we obtain

$$
\underset{k=1}{*+1} x_{k}=x * x_{n+1}=\sum_{v \in V_{2}} c_{v}\left(x, x_{n+1}\right)_{v} .
$$


For any word $v \in V_{2}$, with $v=v_{1} \cdots v_{i}$ for some $i=1, \ldots, S$, we let

$$
|v|_{1}=\operatorname{card}\left\{h \in\{1, \ldots, i\} \mid v_{h}=1\right\} .
$$

Then we define the word $\widehat{v} \in V_{|v|_{1}+1}$ in the following way:

(i) the $|v|_{1}$ indexes 1 in $v$ are progressively relabeled to $1, \ldots,|v|_{1}$;

(ii) the $i-|v|_{1}$ indexes 2 in $v$ are relabeled to $|v|_{1}+1$.

We denote the resulting word by $\widehat{v}$. For example, if $v=11221211$ then $\widehat{v}=12663645$. Using this notation, we have

$$
\left(x, x_{n+1}\right)_{v}=(\underbrace{x, \ldots, x}_{|v|_{1}}, x_{n+1})_{\widehat{v}}=\sum_{w_{1}, \ldots, w_{||_{1}} \in V_{n}} c_{w_{1}} \cdots c_{w_{\mid v_{1}}}\left(x_{w_{1}}, \ldots, x_{w_{|v|}}, x_{n+1}\right)_{\hat{v}} .
$$

By Lemma 3.1, there exist constants $d_{v, w_{1}, \ldots, w_{||_{1}}}^{u} \in\{0, \pm 1\}, u \in V_{n+1}$, such that

$$
\left(x_{w_{1}}, \ldots, x_{w_{|v| 1}}, x_{n+1}\right)_{\widehat{v}}=\sum_{u \in V_{n+1}} d_{v, w_{1}, \ldots, w_{||_{\mid}}}^{u}\left(x_{1}, \ldots, x_{n+1}\right)_{u} .
$$

From $(3 \cdot 11)$ and $(3 \cdot 12)$, we finally obtain

$$
\underset{k=1}{n+1} x_{k}=\sum_{u \in V_{n+1}} c_{u}\left(x_{1}, \ldots, x_{n+1}\right)_{u},
$$

where

$$
c_{u}=\sum_{v \in V_{2}} \sum_{w_{1}, \ldots, w_{|v|} \in V_{n}} c_{v} c_{w_{1}} \cdots c_{w_{|v|} \mid} d_{v, w_{1}, \ldots, w_{|v|_{1}}}^{u} \in A_{n+1} .
$$

In the sum, there are at most $\ell_{n+1}=(n S)^{S}$ summands.

Now we show that $c_{u} \in A_{m}$ for all $u \in V_{n+1}^{m}$ with $2 \leqslant m \leqslant \min \{n+1, S\}$. Assume that in $\left(x_{1}, \ldots, x_{n+1}\right)_{u}$ there appear at most $m \leqslant S$ distinct elements chosen from $x_{1}, \ldots, x_{n+1}$ (if $m>S$ then $\left(x_{1}, \ldots, x_{n+1}\right)_{u}=0$ by (1.6)). From (3.12), we deduce that in the commutator $\left(x_{w_{1}}, \ldots, x_{w_{|v|}}, x_{n+1}\right)_{\widehat{v}}$ there appear at most $m$ distinct elements chosen from $x_{1}, \ldots, x_{n+1}$. On the other hand, $x_{n+1}$ must appear at least once. If this is not the case, then we have either $\left(x, x_{n+1}\right)_{v}=0$ or $v \in W_{1,2}$ and there would be nothing to prove. It follows that in each commutator $x_{w_{1}}, \ldots, x_{w_{|v|}}$ there can appear at most $m-1$ distinct elements chosen from $x_{1}, \ldots, x_{n}$. Then, by induction we have $c_{w_{1}}, \ldots, c_{w_{\mid v_{1}}} \in A_{m-1}$. This shows that $c_{u} \in A_{m}$.

For $i=1, \ldots, S$, let us introduce the ordered pairs of words

$$
G_{i, n}=W_{i, n} \times W_{i, S} \quad \text { and } \quad G_{n}=G_{1, n} \cup \ldots \cup G_{S, n} .
$$

For any pair $(w, k) \in G_{n}$, define

$$
\left(x_{1}, \ldots, x_{n}\right)_{w, k}=\left[x_{w_{1}}^{\left(k_{1}\right)},\left[\ldots\left[x_{w_{i-1}}^{\left(k_{i-1}\right)}, x_{w_{i}}^{\left(k_{i}\right)}\right] \ldots\right]\right] ;
$$

see (1.7) for the notation used here. The first word $w=w_{1} \cdots w_{i}$ of the pair $(w, k)$ in $G_{i, n}$ selects $i$ elements out of $n$ elements $x_{1}, \ldots, x_{n} \in \mathrm{g}$. The second word $k=k_{1} \cdots k_{i}$ selects corresponding strata in the Lie algebra decomposition (1.5). We also define the weight of a word $k \in W_{i, S}$ to be

$$
|k|=k_{1}+\cdots+k_{j} .
$$


COROLlaRY 3.1. Let $x=x_{1} * \cdots * x_{n}$ with $x_{1}, \ldots, x_{n} \in \mathrm{g}$, and $x=x^{(1)}+\cdots+x^{(S)}$ with $x^{(i)} \in \mathrm{g}_{i}$. Then, for any $i=1, \ldots, S$ we have

$$
x^{(i)}=\sum_{\substack{(w, k) \in G_{n} \\|k|=i}} c_{w}\left(x_{1}, \ldots, x_{n}\right)_{w, k} .
$$

Proof. First notice that if $w \in W_{i, n}, i=1, \ldots, S$, then

$$
\left(x_{1}, \ldots, x_{n}\right)_{w}=\sum_{k \in W_{i, S}}\left(x_{1}, \ldots, x_{n}\right)_{w, k} .
$$

Then by Theorem 3.2 we obtain

$$
x=\sum_{w \in V_{n}} c_{w}\left(x_{1}, \ldots, x_{n}\right)_{w}=\sum_{(w, k) \in G_{n}} c_{w}\left(x_{1}, \ldots, x_{n}\right)_{w, k} .
$$

On the other hand, from (1.6) it follows that for all $i, j \geqslant 1$

$$
\left[\mathrm{g}_{i}, \mathrm{~g}_{j}\right] \subset \mathrm{g}_{i+j},
$$

and thus $\left(x_{1}, \ldots, x_{n}\right)_{w, k} \in \mathrm{g}_{i}$ if $|k|=i$. Formula (3.16) follows.

\section{Transition map}

Let us recall that we have identified the group $\mathbb{G}$ and its Lie algebra $g$ via the BakerCampbell-Hausdorff formula. Throughout this section, we write $g$ in place of $\mathbb{G}$ to conform with the notation of the previous section.

Let $a_{1}, \ldots, a_{M} \in \mathrm{g}$ and $r_{1}, \ldots, r_{M}>0$. Throughout this section, we assume that

$$
r_{\text {max }}=\max \left\{r_{1}, \ldots, r_{M}\right\}<\frac{1}{2} .
$$

Let $f_{j}: \mathrm{g} \rightarrow \mathrm{g}$ denote the contraction $f_{j}(x)=a_{j} * \delta_{r_{j}}(x), j=1, \ldots, M$. Denote by $K_{\mathbf{a}}$ the invariant set of $\mathbf{f}=\left\{f_{1}, \ldots, f_{M}\right\}$, where $\mathbf{a}=\left(a_{1}, \ldots, a_{M}\right) \in \mathrm{g}^{M}$ is the $M$-tuple of translation vectors. The projection $\pi_{\mathbf{a}}: W_{\infty, M} \rightarrow K_{\mathbf{a}}$ is

$$
\pi_{\mathbf{a}}(\omega)=\lim _{\ell \rightarrow \infty} f_{\omega_{1}} \circ \cdots \circ f_{\omega_{\ell}}(0) .
$$

For $\omega, v \in W_{\infty, M}$, define the transition map $T_{\omega, v}=\left(T_{\omega, v}^{1}, \ldots, T_{\omega, v}^{M}\right): \mathrm{g}^{M} \rightarrow \mathrm{g}^{M}$

$$
T_{\omega, v}^{j}(\mathbf{a})= \begin{cases}\pi_{\mathbf{a}}(\omega)^{-1} * \pi_{\mathbf{a}}(v) & \text { for } j=1 \\ a_{i} & \text { for } j=2, \ldots, M .\end{cases}
$$

In what follows, we make use of words in $W_{\infty, M}$ indexed by words chosen from another symbol set $W_{i, \infty}$. For this reason, in the rest of this section we denote elements of $W_{\infty, M}$ by Greek letters.

The aim of this section is to prove the following theorem.

THEOREM $4 \cdot 1$ (Euclidean bi-Lipschitz continuity of the transition map). Let $\omega, v$ be elements of $W_{\infty, M}$ satisfying $1=\omega_{1} \neq v_{1}$. For any compact set $K \subset \mathrm{g}^{M}$ there exists a constant $C=C(K) \geqslant 1$ independent of $\omega$ and $v$ such that $\left.T_{\omega, v}\right|_{K}$ is C-bi-Lipschitz continuous on $K$ with respect to the standard metric. 
By standard metric, we mean any metric on the vector space $\mathrm{g}^{M}$ which is induced by a norm, e.g., the Euclidean metric. Under the aforementioned identification of $g$ and $\mathbb{G}$, this corresponds to the Euclidean metric on $\mathbb{G}^{M}$.

\subsection{Projection map}

Our first task is to compute an explicit formula for the mapping $\pi_{\mathbf{a}}$. Recall that, according to $(3 \cdot 14)$, we have

$$
G_{M}=\bigcup_{i=1}^{S} W_{i, M} \times W_{i, S} .
$$

We use the following notation. For $(g, k) \in W_{i, M} \times W_{i, S}$ we let

$$
\mathbf{a}_{g, k}=\left[a_{g_{1}}^{\left(k_{1}\right)},\left[\ldots,\left[a_{g_{i-1}}^{\left(k_{i-1}\right)}, a_{g_{i}}^{\left(k_{i}\right)}\right] \ldots\right]\right] .
$$

This is consistent with (3.15). Moreover, for $\omega \in W_{\infty, M}, v \in W_{i, \infty}$ and $k \in W_{i, S}$ we let

$$
\mathbf{a}_{v, k}(\omega)=\left[a_{\omega_{v_{1}}}^{\left(k_{1}\right)},\left[\ldots,\left[a_{\omega_{v_{i-1}}}^{\left(k_{i-1}\right)}, a_{\omega_{v_{i}}}^{\left(k_{i}\right)}\right] \ldots\right]\right] .
$$

For any $g \in W_{i, M}$ and $\omega \in W_{\infty, M}$, let $W_{g}(\omega) \subset W_{i, \infty}$ denote the set of all $v \in W_{i, \infty}$ such that

$$
\left(a_{\omega_{v_{1}}}, \ldots, a_{\omega_{v_{i}}}\right)=\left(a_{g_{1}}, \ldots, a_{g_{i}}\right) .
$$

Finally, let $r_{\omega_{1} \cdots \omega_{n}}=r_{\omega_{1}} \cdots r_{\omega_{n}}$, where we stipulate that the empty product is equal to one. For $\omega \in W_{\infty, M}, v \in W_{i, \infty}$ and $k \in W_{i, S}$ let

$$
r_{v, k}(\omega):=\prod_{h=1}^{i} r_{\omega_{1} \ldots \omega_{v_{h}-1}}^{k_{k}} .
$$

LEMMA $4 \cdot 1$ (Representation Formula for $\pi_{\mathbf{a}}$ ). For any $\omega \in W_{\infty, M}$ we have

$$
\pi_{\mathbf{a}}(\omega)=\sum_{(g, k) \in G_{M}} \gamma_{g, k}(\omega) \mathbf{a}_{g, k},
$$

where the coefficients $\gamma_{g, k}(\omega) \in \mathbb{R}$ are given by

$$
\gamma_{g, k}(\omega)=\sum_{v \in W_{g}(\omega)} c_{v} r_{v, k}(\omega) .
$$

Here, the constants $c_{v} \in \mathbb{R}$ are given by Theorem $3 \cdot 2$.

Proof. We prove by induction on $n \in \mathbb{N}$ that for any $\omega \in W_{\infty, M}$ and $x \in \mathrm{g}$ we have

$$
f_{\omega_{1}} \circ \cdots \circ f_{\omega_{n}}(x)=\underset{k=1}{*} \delta_{r_{\omega_{1} \cdots \omega_{k-1}}}\left(a_{\omega_{k}}\right) * \delta_{r_{\omega_{1} \ldots \omega_{n}}}(x) .
$$

For $n=1$ this is the definition of $f_{\omega_{1}}$. Using the properties $\delta_{\varrho}\left(\delta_{\sigma}(a)\right)=\delta_{\varrho \sigma}(a)$ and $\delta_{\varrho}(a) *$ $\delta_{\varrho}(b)=\delta_{\varrho}(a * b)$, we get

$$
\begin{aligned}
f_{\omega_{1}} \circ \cdots \circ f_{\omega_{n+1}}(x) & =f_{\omega_{1}} \circ \cdots \circ f_{\omega_{n}}\left(f_{\omega_{n+1}}(x)\right) \\
& =\underset{k=1}{*} \delta_{r_{\omega_{1} \cdots \omega_{k-1}}}\left(a_{\omega_{k}}\right) * \delta_{r_{\omega_{1} \cdots \omega_{n}}}\left(a_{\omega_{n+1}} * \delta_{r_{\omega_{n+1}}}(x)\right) \\
& =\underset{k=1}{n+1} \delta_{r_{\omega_{1} \cdots \omega_{k-1}}}\left(a_{\omega_{k}}\right) * \delta_{r_{\omega_{1} \cdots \omega_{n+1}}}(x) .
\end{aligned}
$$


This is (4.9) for $n+1$. Choosing $x=0$ in (4.9), we obtain

$$
x_{n}:=f_{\omega_{1}} \circ \ldots \circ f_{\omega_{n}}(0)=\underset{k=1}{*} \delta_{r_{\omega_{1} \cdots \omega_{k-1}}}\left(a_{\omega_{k}}\right) .
$$

Using the notation (4.6) and (4.4) and beginning from (3.16), we obtain

$$
x_{n}=\sum_{(v, k) \in G_{n}} c_{v} r_{v, k}(\omega) \mathbf{a}_{v, k}(\omega),
$$

where we also used the property $\delta_{r_{\omega_{1} \cdots \omega_{k-1}}}\left(a_{\omega_{k}}\right)^{(i)}=r_{\omega_{1} \cdots \omega_{k-1}}^{i} a_{\omega_{k}}^{(i)}, i=1, \ldots, S$. Formula (4·10) can be rearranged in the following way

$$
x_{n}=\sum_{(g, k) \in G_{M}} \gamma_{g, k}^{n}(\omega) \mathbf{a}_{g, k},
$$

where the coefficients $\gamma_{g, k}^{n}(\omega) \in \mathbb{R}$ are given by

$$
\gamma_{g, k}^{n}(\omega)=\sum_{v \in W_{g}^{n}(\omega)} c_{v} r_{v, k}(\omega)
$$

Here $W_{g}^{n}(\omega)$ is the set of $v \in W_{i, n}$ for which (4.5) holds. Letting $n \rightarrow \infty$ yields (4.7).

LEMMA 4.2. There exists a constant $C_{1}<\infty$ such that

$$
\left|\gamma_{g, k}(\omega)\right| \leqslant C_{1}
$$

for all $(g, k) \in G_{M}$ and $\omega \in W_{\infty, M}$.

Proof. For $n \in \mathbb{N}$ and $(g, k) \in G_{M}$ we have

$$
\left|\gamma_{g, k}^{n}(\omega)\right|=\left|\sum_{v \in W_{g}^{n}(\omega)} c_{v} r_{v, k}(\omega)\right| \leqslant \max _{c_{w} \in A}\left|c_{w}\right| \sum_{v \in W_{g}^{n}(\omega)} r_{v, k}(\omega),
$$

where $A \subset \mathbb{Q}$ is the finite set given by Theorem 3.2. For $(v, k) \in G_{i, n}, i=1, \ldots, S$, using the formula (4.6) we have

$$
r_{v, k}(\omega)=\prod_{h=1}^{i} r_{\omega_{1} \cdots \omega_{v_{h}-1}}^{k_{k}} \leqslant \prod_{h=1}^{i}\left(\frac{1}{2}\right)^{v_{h}-1}
$$

since $r_{1}, \ldots, r_{M}<1 / 2$ and $k_{1}, \ldots, k_{i} \geqslant 1$. Replacing the sum over $v \in W_{g}^{n}(\omega)$ with the sum over all $v \in W_{i, n}$, for the appropriate choice of $i \in\{1, \ldots, S\}$, we obtain

$$
\sum_{v \in W_{g}^{n}(\omega)} r_{v, k}(\omega) \leqslant \sum_{v_{1}, \ldots, v_{i}=1}^{n} \prod_{h=1}^{i}\left(\frac{1}{2}\right)^{v_{h}-1}=\left(\sum_{v=1}^{n} 2^{1-v}\right)^{i} \leqslant 2^{i} \leqslant 2^{S} .
$$

Then (4.11) holds with $C=2^{S} \max _{c_{w} \in A}\left|c_{w}\right|$.

\subsection{Formula for the transition map}

Next, we provide an explicit formula for the transition map $T_{\omega, v}$. Let $i \in\{1, \ldots, S\}$. For pairs $\left(g_{1}, k_{1}\right), \ldots,\left(g_{i}, k_{i}\right)$ in $G_{M}$, let

$$
\mathbf{a}_{g_{1}, k_{1}, \ldots, g_{i}, k_{i}}=\left[\mathbf{a}_{g_{1}, k_{1}},\left[\ldots\left[\mathbf{a}_{g_{i-1}, k_{i-1}}, \mathbf{a}_{g_{i}, k_{i}}\right] \ldots\right]\right] .
$$


LEMmA 4.3 (Representation Formula for the transition map). For $\omega, v \in W_{\infty, M}$ we have

$$
T_{\omega, v}^{1}(\mathbf{a})=\sum_{i=1}^{S} \sum_{\left(g_{1}, k_{1}\right), \ldots,\left(g_{i}, k_{i}\right) \in G_{M}} \Gamma_{g_{1}, k_{1}, \ldots, g_{i}, k_{i}}(\omega, v) \mathbf{a}_{g_{1}, k_{1}, \ldots, g_{i}, k_{i}},
$$

where the coefficients $\Gamma_{g_{1}, k_{1}, \ldots, g_{i}, k_{i}}(\omega, v) \in \mathbb{R}$ are given by

$$
\Gamma_{g_{1}, k_{1}, \ldots, g_{i}, k_{i}}(\omega, v)=\sum_{u \in W_{i, 2}} c_{u} \prod_{h=1}^{i}(-1)^{u_{h}} \gamma_{g_{h}, k_{h}}\left(\omega^{u_{h}}\right), \quad \omega^{u_{h}}= \begin{cases}\omega, & u_{h}=1, \\ v, & u_{h}=2 .\end{cases}
$$

Proof. Let $x_{1}=\pi_{\mathbf{a}}(\omega)^{-1}=-\pi_{\mathbf{a}}(\omega)$ and $x_{2}=\pi_{\mathbf{a}}(v)$. From (3.2) we get

$$
T_{\omega, v}^{1}(\mathbf{a})=\sum_{i=1}^{S} \sum_{u \in W_{i, 2}} c_{u}\left(x_{1}, x_{2}\right)_{u}
$$

and, using (4.7), we find

$$
\begin{aligned}
\left(x_{1}, x_{2}\right)_{u} & =\left[x_{u_{1}},\left[\ldots,\left[x_{u_{i-1}}, x_{u_{i}}\right] \ldots\right]\right] \\
& =\sum_{\left(g_{1}, k_{1}\right), \ldots,\left(g_{i}, k_{i}\right) \in G_{M}} \prod_{h=1}^{i}(-1)^{u_{h}} \gamma_{g_{h}, k_{h}}\left(\omega^{u_{h}}\right) \mathbf{a}_{g_{1}, k_{1}, \ldots, g_{i}, k_{i}} .
\end{aligned}
$$

The formulas (4.13) and (4.14) follow.

LEMmA 4.4. There exists a constant $C_{2}<\infty$ such that

$$
\left|\Gamma_{g_{1}, k_{1}, \ldots, g_{i}, k_{i}}(\omega, v)\right| \leqslant C_{2}
$$

for all $\left(g_{1}, k_{1}\right), \ldots,\left(g_{i}, k_{i}\right) \in G_{M}, i=1, \ldots, S$, and all $\omega, v \in W_{\infty, M}$.

Proof. By (4.14), we have

$$
\left|\Gamma_{g_{1}, k_{1}, \ldots, g_{i}, k_{i}}(\omega, v)\right| \leqslant \sum_{u \in W_{i, 2}}\left|c_{u}\right| \prod_{h=1}^{i}\left|\gamma_{g_{h}, k_{h}}\left(\omega^{u_{h}}\right)\right| .
$$

Now (4-15) follows from (4.11) with $C_{2}=2{ }^{S} C_{1}^{S} \max _{c_{u} \in A}\left|c_{u}\right|$.

LeMmA 4.5. Let $\omega, v \in W_{\infty, M}$ with $\omega_{1} \neq v_{1}$. Then

$$
\left|\Gamma_{g, s}(\omega, v)\right| \geqslant \frac{1-2 r_{\max }^{i}}{1-r_{\max }^{i}}
$$

for all $g \in\left\{\omega_{1}, v_{1}\right\}$ and $i=1, \ldots, S$.

Proof. By (4.14), (4.8), (4.6) and the triangle inequality, we obtain

$$
\begin{aligned}
\left|\Gamma_{g, i}(\omega, v)\right| & =\left|\gamma_{g, i}(v)-\gamma_{g, i}(\omega)\right| \\
& =\left|\sum_{u \in W_{g}(v)} c_{u} r_{u, i}(v)-\sum_{u \in W_{g}(\omega)} c_{u} r_{u, i}(\omega)\right| \\
& =\left|\sum_{u=1}^{\infty} r_{v_{1} \cdots v_{u-1}}^{i} \delta_{v_{u} g}-\sum_{u=1}^{\infty} r_{\omega_{1} \cdots \omega_{u-1}}^{i} \delta_{\omega_{u} g}\right| \\
& \geqslant 1-\left|\sum_{u=2}^{\infty} r_{v_{1} \cdots v_{u-1}}^{i} \delta_{v_{u} g}-\sum_{u=2}^{\infty} r_{\omega_{1} \cdots \omega_{u-1}}^{i} \delta_{\omega_{u} g}\right| .
\end{aligned}
$$


Here we wrote $\delta_{v w}$ for the Kronecker delta function. We estimate

$$
\begin{aligned}
\left|\sum_{u=2}^{\infty} r_{v_{1} \cdots v_{u-1}}^{i} \delta_{v_{u} g}-\sum_{u=2}^{\infty} r_{\omega_{1} \cdots \omega_{u-1}}^{i} \delta_{\omega_{u} g}\right| & \leqslant \sum_{u=1}^{\infty} \max \left\{r_{v_{1} \ldots v_{u}}^{i}, r_{\omega_{1} \ldots \omega_{u}}^{i}\right\} \\
& \leqslant \sum_{u=1}^{\infty} r_{\max }^{i u}=\frac{r_{\max }^{i}}{1-r_{\max }^{i}}
\end{aligned}
$$

which is strictly less than one since $i \geqslant 1$ and $r_{\max }<1 / 2$. Estimate (4.16) follows.

Remark 4.1. The careful reader will note that, in contrast with the results of [22] and [8], the proof of (4.16) does not require Solomyak's trick [22] to obtain the desired esimate under the assumption $r_{\max }<1 / 2$. The reason is that we work throughout this paper only with homotheties $f: \mathbb{G} \rightarrow \mathbb{G}, f=\tau_{a} \circ \delta_{r}, a \in \mathbb{G}, r>0$, i.e., we do not allow any rotations in the defining mappings. This simplifies the work involved in obtaining the estimate in (4.18), since the two terms on the left hand side cannot contribute simultaneously with the same sign.

Let us split the mapping $T_{\omega, v}^{1}$ into its linear and nonlinear parts. The linear part can be determined by setting $i=1$ in (4.13) and choosing $g \in W_{1, M}, k \in W_{1, S}$ in (4.3). Namely, the linear part is

$$
\bar{T}_{\omega, v}^{1}(\mathbf{a})=\sum_{(g, k) \in W_{1, M} \times W_{1, S}} \Gamma_{g, k}(\omega, v) \mathbf{a}_{g, k}=\sum_{g=1}^{M} \sum_{k=1}^{S} \Gamma_{g, k}(\omega, v) a_{g}^{(k)},
$$

where

$$
\Gamma_{g, k}(\omega, v)=\sum_{u \in W_{1,2}} c_{u}(-1)^{u} \gamma_{g, k}\left(\omega^{u}\right)=\gamma_{g, k}(v)-\gamma_{g, k}(\omega) .
$$

The nonlinear part is $\widehat{T}_{\omega, v}^{1}=T_{\omega, v}^{1}-\bar{T}_{\omega, v}^{1}$. We have the decomposition

$$
T_{\omega, v}^{1}=\bar{T}_{\omega, v}^{1}+\widehat{T}_{\omega, v}^{1} .
$$

The elements $\mathbf{a}=\left(a_{1}, \ldots, a_{M}\right) \in \mathrm{g}^{M}$ and $T_{\omega, v}^{1}(\mathbf{a}) \in \mathrm{g}$ decompose as

$$
\mathbf{a}=\mathbf{a}^{(1)}+\cdots+\mathbf{a}^{(S)}, \quad \mathbf{a}^{(i)}=\left(a_{1}^{(i)}, \ldots, a_{M}^{(i)}\right),
$$

and

$$
T_{\omega, v}^{1}(\mathbf{a})=T_{\omega, v}^{1}(\mathbf{a})^{(1)}+\cdots+T_{\omega, v}^{1}(\mathbf{a})^{(S)} .
$$

We have the relation

$$
T_{\omega, v}^{1}(\mathbf{a})^{(1)}=\bar{T}_{\omega, v}^{1}\left(\mathbf{a}^{(1)}\right) .
$$

By (1.6), the variables $\mathbf{a}^{(h)}=\left(a_{1}^{(h)}, \ldots, a_{M}^{(h)}\right)$ with $h \geqslant i$ do not occur in $\widehat{T}_{\omega, v}^{1}(\mathbf{a})^{(i)}$. Hence we also have the relation

$$
T_{\omega, v}^{1}(\mathbf{a})^{(i)}=\bar{T}_{\omega, v}^{1}\left(\mathbf{a}^{(i)}\right)+\widehat{T}_{\omega, v}^{1}\left(\mathbf{a}^{(1)}+\cdots+\mathbf{a}^{(i-1)}\right)^{(i)}
$$

for $i=2, \ldots, S$.

Lemma 4.6. Let $\omega, v \in W_{\infty, M}$ satisfy $1=\omega_{1} \neq v_{1}$. Then the map $T_{\omega, v}: \mathrm{g}^{M} \rightarrow \mathrm{g}^{M}$ is surjective. 
Proof. For $\mathbf{b}=\left(b_{1}, \ldots, b_{M}\right) \in \mathrm{g}^{M}$, we look for $\mathbf{a}=\left(a_{1}, \ldots, a_{M}\right) \in \mathrm{g}^{M}$ such that $T_{\omega, v}(\mathbf{a})=\mathbf{b}$, i.e.

$$
\begin{aligned}
T_{\omega, v}^{1}\left(a_{1}, \ldots, a_{M}\right) & =b_{1} \\
a_{2} & =b_{2} \\
& \vdots \\
a_{M} & =b_{M},
\end{aligned}
$$

or, in fact,

$$
T_{\omega, v}^{1}\left(a_{1}, b_{2}, \ldots, b_{M}\right)=b_{1} .
$$

We solve the equation (4.23) layer by layer. In the first layer, we have

$$
\bar{T}_{\omega, v}^{1}\left(a_{1}^{(1)}, b_{2}^{(1)}, \ldots, b_{M}^{(1)}\right)=T_{\omega, v}^{1}\left(a_{1}, b_{2}, \ldots, b_{M}\right)^{(1)}=b_{1}^{(1)},
$$

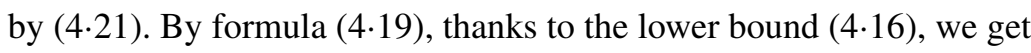

$$
a_{1}^{(1)}=\frac{1}{\Gamma_{1,1}(v, \omega)} b_{1}^{(1)}-\sum_{g=2}^{M} \frac{\Gamma_{g, 1}(v, \omega)}{\Gamma_{1,1}(v, \omega)} b_{g}^{(1)}:=\Theta_{\omega, v}^{1}(\mathbf{b})^{(1)} .
$$

Next, we solve equation (4.23) in the second layer. By (4.22) we have

$$
\begin{aligned}
T_{\omega, v}^{1}\left(a_{1}, b_{2}, \ldots, b_{M}\right)^{(2)} & =\bar{T}_{\omega \omega, v}^{1}\left(a_{1}^{(2)}, b_{2}^{(2)}, \ldots, b_{M}^{(2)}\right)+\widehat{T}_{\omega, v}^{1}\left(\Theta_{\omega, v}^{1}(\mathbf{b})^{(1)}, b_{2}^{(1)}, \ldots, b_{M}^{(1)}\right)^{(2)} \\
& =b_{1}^{(2)} .
\end{aligned}
$$

Using again (4.19) and the lower bound (4.16), we get

$$
\begin{aligned}
a_{1}^{(2)} & =\frac{1}{\Gamma_{1,2}(v, \omega)}\left\{b_{1}^{(2)}-\sum_{g=2}^{M} \Gamma_{g, 2}(v, \omega) b_{g}^{(2)}-\widehat{T}_{\omega, v}^{1}\left(\Theta_{\omega, v}^{1}(\mathbf{b})^{(1)}, b_{2}^{(1)}, \ldots, b_{M}^{(1)}\right)^{(2)}\right\} \\
: & =\Theta_{\omega, v}^{1}(\mathbf{b})^{(2)} .
\end{aligned}
$$

Now $a_{1}^{(i)}=\Theta_{\omega, v}^{1}(\mathbf{b})^{(i)}$ can be determined recursively for each $i=1, \ldots, S$. In fact,

$$
a_{1}^{(i)}=\frac{1}{\Gamma_{1, i}(v, \omega)}\left\{b_{1}^{(i)}-\sum_{g=2}^{M} \Gamma_{g, 2}(v, \omega) b_{g}^{(i)}-\Lambda_{\omega, v}^{(i)}(\mathbf{b})\right\}:=\Theta_{\omega, v}^{1}(\mathbf{b})^{(i)},
$$

where

$$
\Lambda_{\omega, v}^{(i)}(\mathbf{b})=\widehat{T}_{\omega, v}^{1}\left(\Theta_{\omega, v}^{1}(\mathbf{b})^{(1)}+\cdots+\Theta_{\omega, v}^{1}(\mathbf{b})^{(i-1)}, b_{2}^{(1)}+\cdots+b_{2}^{(i-1)}, \ldots, b_{M}^{(1)}+\cdots+b_{M}^{(i-1)}\right)^{(i)} .
$$

This completes the proof.

The proof of Lemma 4.6 provides a formula for the inverse $\Theta_{\omega, v}: \mathrm{g}^{M} \rightarrow \mathrm{g}^{M}$ of the mapping $T_{\omega, v}$. In fact, the $i$ th component of $\Theta_{\omega, v}$ is

$$
\Theta_{\omega, v}^{j}(\mathbf{b})= \begin{cases}\Theta_{\omega, v}^{1}(\mathbf{b})^{(1)}+\cdots+\Theta_{\omega, v}^{1}(\mathbf{b})^{(S)} & \text { for } j=1, \\ b_{i} & \text { for } j=2, \ldots, M,\end{cases}
$$

where $\Theta_{\omega, v}^{1}(\mathbf{b})^{(i)}$ is given by (4.24).

Proof of Theorem 4.1. For any $\left(g_{1}, k_{1}\right), \ldots,\left(g_{i}, k_{i}\right) \in G_{M}$, the map a $\mapsto \mathbf{a}_{g_{1}, k_{1}, \ldots, g_{i}, k_{i}}$ is locally Lipschitz continuous from $\mathrm{g}^{M}$ to $\mathrm{g}$. According to Proposition 4.3, the map 
$T_{\omega, v}^{1}: \mathrm{g}^{M} \rightarrow \mathrm{g}$ is thus a linear combination of locally Lipschitz continuous mappings. By Lemma 4.4, the coefficients in this linear combination are bounded by a constant which is independent of the words $\omega$ and $v$. The map $T_{\omega, v}$ is then Lipschitz continuous on compact sets, with a Lipschitz constant independent of the words.

An inspection of (4.24) and the bounds given by Lemmas 4.4 and 4.5 show that the inverse map $\Theta_{\omega, v}$ is also locally Lipschitz continuous with Lipschitz constant which is independent of the words $\omega$ and $v$. This claim can be formally proved by induction on $i=1, \ldots, S$.

\section{Proof of the main theorem}

\subsection{Dimension comparison in Carnot groups}

Let $\mathbb{G}$ denote a Carnot group equipped with a sub-Riemannian metric $d$. As mentioned in the introduction, the metric structure of $(\mathbb{G}, d)$ differs substantially from the metric structure of the underlying Euclidean space $\mathbb{R}^{N}$. A precise description of the discrepancy between the Hausdorff dimensions of subsets of $\mathbb{G}$ measured with respect to sub-Riemannian and Euclidean metrics was given in [5]. This discrepancy is quantified by the dimension comparison functions $\beta_{ \pm}:[0, N] \rightarrow[0, Q]$ associated to the group $\mathbb{G}$.

Recall that we have denoted by $m_{i}$ the dimension of the $i$ th layer $\mathrm{g}_{i}$ of the Lie algebra $\mathrm{g}$ of $\mathbb{G}$. The lower dimension comparison function for $\mathbb{G}$ is the function $\beta_{-}=\beta_{-}^{\mathbb{G}}:[0, N] \rightarrow$ $[0, Q]$ defined by

$$
\beta_{-}(\alpha)=\sum_{i=1}^{\ell_{-}} i m_{i}+\left(1+\ell_{-}\right)\left(\alpha-\sum_{i=1}^{\ell_{-}} m_{i}\right),
$$

where $\ell_{-}=\ell_{-}(\alpha) \in\{0, \ldots, S-1\}$ is the unique integer satisfying

$$
\sum_{i=1}^{\ell_{-}} m_{i}<\alpha \leqslant \sum_{i=1}^{1+\ell_{-}} m_{i}
$$

Similarly, the upper dimension comparison function for $\mathbb{G}$ is the function $\beta_{+}=\beta_{+}^{\mathbb{G}}$ : $[0, N] \rightarrow[0, Q]$ defined by

$$
\beta_{+}(\alpha)=\sum_{i=\ell_{+}}^{S} i m_{i}+\left(-1+\ell_{+}\right)\left(\alpha-\sum_{i=\ell_{+}}^{S} m_{i}\right),
$$

where $\ell_{+}=\ell_{+}(\alpha) \in\{2, \ldots, S+1\}$ is the unique integer satisfying

$$
\sum_{i=\ell_{+}}^{S} m_{i}<\alpha \leqslant \sum_{i=-1+\ell_{+}}^{S} m_{i}
$$

Here we stipulate that the empty sum is equal to zero.

Intuitively, $\beta_{-}(\alpha)$ is a weighted sum of dimensions of layers of $g$, starting from the lowest layer $g_{1}$ and increasing up to the layer $g_{\ell_{-}(\alpha)}$, together with a portion of the layer $g_{1+\ell_{-}(\alpha)}$, while $\beta_{+}(\alpha)$ is a weighted sum of dimensions of layers of $\mathrm{g}$, starting from the highest layer $\mathrm{g}_{S}$ and decreasing down to the layer $\mathrm{g}_{\ell_{+}(\alpha)}$, together with a portion of the layer $\mathrm{g}_{-1+\ell_{+}(\alpha)}$.

The relevance of these dimension comparison functions is evident in [5, theorems 2.4 and 2.6]: For any set $A \subset \mathbb{G}, \beta_{-}\left(\operatorname{dim}_{E} A\right) \leqslant \operatorname{dim}_{c c} A \leqslant \beta_{+}\left(\operatorname{dim}_{E} A\right)$ and every pair $(\alpha, \beta)$ with $\beta_{-}(\alpha) \leqslant \beta \leqslant \beta_{+}(\alpha)$ coincides with $\left(\operatorname{dim}_{E} A, \operatorname{dim}_{c c} A\right)$ for some $A \subset \mathbb{G}$. 
For later purposes we provide an explicit formula for the lower dimension comparison function. For $s \in[0, Q]$ we have

$$
\begin{aligned}
\beta_{-}^{-1}(s) & =\min _{i=1, \ldots, s}\left(\frac{\sum_{h=1}^{i}(i-h) m_{h}+s}{i}\right) \\
& =\min \left\{s, \frac{m_{1}+s}{2}, \frac{2 m_{1}+m_{2}+s}{3}, \ldots, \frac{S N-Q+s}{S}\right\} .
\end{aligned}
$$

\subsection{A covering lemma}

Let $T_{\omega, v}: \mathbb{G}^{M} \rightarrow \mathbb{G}^{M}$ be the mapping introduced in (4.2). By Theorem $4 \cdot 1, T_{\omega, v}$ is bi-Lipschitz continuous on compact sets in the Euclidean metric, with a Lipschitz constant independent of the words $\omega$ and $v$.

We fix a parameter $R \geqslant 1$. For $\varrho \leqslant 1$ we introduce the set

$$
\Omega_{\omega, v}(\varrho)=\left(T_{\omega, v}^{-1}\right)\left(B_{\varrho} \times B_{R}^{M-1}\right) .
$$

LEMMA 5·1. Let $(\mathbb{G}, d)$ be a Carnot group of step $S$ equipped with a sub-Riemannian metric. Consider the product Carnot group $\mathbb{G}^{M}=(\mathbb{G}, d)^{M}$. For each $i=1, \ldots, S$, the set $\Omega_{\omega, v}(\varrho) \subset \mathbb{G}^{M}$ can be covered by at most

$$
C \varrho^{-i(Q M-N)-\sum_{h=1}^{i}(i-h) m_{h}}
$$

balls of radius $\varrho^{i}$, where $C=C(\mathbb{G}, R)$.

In the proof of Lemma $5 \cdot 1$, we make use of the following auxiliary covering lemma.

LEMMA $5 \cdot 2$ (Balogh-Tyson-Warhurst). Let $K \subset \mathbb{G}$ be a bounded set.

(a) For each $h=1, \ldots, S$, each Euclidean ball of radius $r$ with center in $K$ can be covered by $O\left(r^{-\lambda_{+}(h+1)}\right)$ balls of radius $r^{1 / h}$, where

$$
\lambda_{+}(h+1)=\sum_{i=h}^{S}\left(\frac{i}{h}-1\right) m_{i} .
$$

(b) For each $h=1, \ldots, S$, each ball of radius $r$ with center in $K$ can be covered by $O\left(r^{-\lambda_{-}(h-1)}\right)$ Euclidean balls of radius $r^{h}$, where

$$
\lambda_{-}(h-1)=\sum_{i=1}^{h}(h-i) m_{i} .
$$

The implicit constants depend on $\mathbb{G}$ and $K$, but are independent of $r$.

See [5, lemma 3·2].

The idea of the proof of Lemma $5 \cdot 1$ is quite simple. We will produce the desired covering in a sequence of steps. First, we cover each of the balls $B_{\varrho}$ and $B_{R}$ with families of balls of smaller radii $\varrho^{i / k}$ and $\varrho^{i / \ell}$ respectively. Here $k \in\{1, \ldots, i\}$ and $\ell \in\{1, \ldots, S\}$ are free parameters which will be chosen later in order to minimize the total cardinality of the covering. We then apply Lemma $5 \cdot 2$ to cover each of these balls with Euclidean balls of radii $\varrho^{i}$. By this scheme we produce a covering of $B_{\varrho} \times B_{R}^{M-1}$ by Euclidean balls of radius $\varrho^{i}$. Since the map $T_{\omega, v}^{-1}$ is Euclidean bi-Lipschitz, we obtain a covering of $\Omega_{\omega, v}(\varrho)$ by such Euclidean balls as well. Finally, we use Lemma 5.2 again to convert this Euclidean covering 
in the cardinality of the covering at each stage, and eventually minimize over $k$ and $\ell$. This yields the desired estimate.

Proof of Lemma 5.1. For fixed $\kappa \geqslant 0$, we write $O\left(\varrho^{\kappa}\right)$ to denote any quantity which is bounded above by $C \varrho^{\kappa}$ for some constant $C$ which depends only on the group $\mathbb{G}$ and the radius $R$.

(i) By a simple volume estimate, we may cover $B_{\varrho}$ with

$$
O\left(\left(\frac{\varrho}{\varrho^{i / k}}\right)^{Q}\right)=O\left(\varrho^{Q(1-i / k)}\right)
$$

balls of radius $\varrho^{i / k}$, for any fixed $k \in\{1, \ldots, i\}$. Applying Lemma 5.2(b) with $h=k$ yields a covering of $B_{\varrho}$ by

$$
O\left(\varrho^{Q(1-i / k)-(i / k) \lambda_{-}(k-1)}\right)
$$

Euclidean balls of radius $\varrho^{i}$.

(ii) Similarly, we may cover $B_{R}$ with $O\left(\varrho^{-Q i / \ell}\right)$ balls of radius $\varrho^{i / \ell}$, for any fixed $\ell \in$ $\{1, \ldots, S\}$. Another application of Lemma 5.2(b), this time with $h=\ell$, yields a covering of $B_{R}$ by

$$
O\left(\varrho^{-Q i / \ell-(i / \ell) \lambda_{-}(\ell-1)}\right)
$$

Euclidean balls of radius $\varrho^{i}$.

(iii) Combining (i) and (ii), we find that $B_{\varrho} \times B_{R}^{M-1}$ can be covered by

$$
O\left(\varrho^{Q(1-i / k)-(i / k) \lambda_{-}(k-1)-(M-1) Q i / \ell-(M-1)(i / \ell) \lambda_{-}(\ell-1)}\right)
$$

Euclidean balls of radius $\varrho^{i}$. (Here we are in the space $\mathbb{G}^{M}$.)

(iv) We are now in a position to use the fact that $T_{\omega, v}$ is Euclidean bi-Lipschitz. We conclude that $\Omega_{\omega, v}(\varrho)=T_{\omega, v}^{-1}\left(B_{\varrho} \times B_{R}^{M-1}\right)$ can be covered by

$$
O\left(\varrho^{Q(1-i / k)-(i / k) \lambda_{-}(k-1)-(M-1) Q i / \ell-(M-1)(i / \ell) \lambda_{-}(\ell-1)}\right)
$$

Euclidean balls of radius $\varrho^{i}$.

(v) To conclude the proof we return to the sub-Riemannian metric $d$. By a final application of Lemma 5.2(a) with $h=1$, we cover $\Omega_{\omega, v}(\varrho)$ by

$$
O\left(\varrho^{Q(1-i / k)-(i / k) \lambda_{-}(k-1)-(M-1) Q i / \ell-(M-1)(i / \ell) \lambda_{-}(\ell-1)-M i \lambda_{+}(2)}\right)
$$

Carnot-Carathéodory balls of radius $\varrho^{i}$.

To complete the proof we must evaluate the exponent

$$
Q\left(1-\frac{i}{k}\right)-\frac{i}{k} \lambda_{-}(k-1)-(M-1) Q \frac{i}{\ell}-(M-1) \frac{i}{\ell} \lambda_{-}(\ell-1)-M i \lambda_{+}(2) \text {. }
$$

To this end, we observe that $\lambda_{+}(2)=Q-N$. We may rewrite (5.6) as

$$
-i M(Q-N)+Q-i\left(\frac{Q+\lambda_{-}(k-1)}{k}+(M-1) \frac{Q+\lambda_{-}(\ell-1)}{\ell}\right) .
$$

We minimize over $k \in\{1, \ldots, i\}$ and $\ell \in\{1, \ldots, S\}$. A simple computation shows that the function $k \mapsto\left(Q+\lambda_{-}(k-1)\right) / k$ is decreasing. Thus the minimum occurs for $k=i$ and $\ell=S$. Since $\lambda_{-}(S-1)=S N-Q,(5 \cdot 7)$ reduces to

$$
-i M(Q-N)-\lambda_{-}(i-1)-i(M-1) N=-i(M Q-N)-\lambda_{-}(i-1),
$$




\subsection{Proof of Theorem 1·1.}

Assume that

$$
\operatorname{dim}_{c c} E(s)>Q M-\left(N-\beta_{-}^{-1}(s)\right)
$$

Choose $q$ satisfying

$$
Q M-\left(N-\beta_{-}^{-1}(s)\right)<q<\operatorname{dim}_{c c} E(s) ;
$$

then $\mathcal{H}_{c c}^{q}(E(s))=\infty$, hence $\mathcal{H}_{c c}^{q}\left(E(s) \cap B_{R}^{M}\right)>0$ for some $R>0$. By Frostman's theorem, there exists a nontrivial Borel measure $\mu$, supported on $E(s) \cap B_{R}^{M}$ so that

$$
\mu\left(B_{\varrho}(\mathbf{x})\right) \leqslant \varrho^{q}
$$

for all $\mathbf{x} \in \mathbb{G}^{M}$ and all $\varrho>0$. Thus

$$
\operatorname{dim}_{c c}\left(K_{\mathbf{a}}\right)<s
$$

for $\mu$-a.e. $\mathbf{a} \in B_{R}^{M}$. We shall verify (2.6) for this measure $\mu$; by Proposition $2 \cdot 2$ this inequality implies that

$$
\operatorname{dim}_{c c}\left(K_{\mathbf{a}}\right) \geqslant s
$$

for $\mu$-a.e. $\mathbf{a} \in B_{R}^{M}$ which contradicts (5·10).

The desired estimate

$$
\int_{B_{R}^{M}} d\left(\pi_{\mathbf{a}}(v), \pi_{\mathbf{a}}(\omega)\right)^{-s} d \mu(\mathbf{a}) \leqslant \frac{C(\mathbb{G}, R)}{r_{\omega \wedge v}^{s}}
$$

for all $\omega, v \in W_{\infty, M}$ is equivalent to the estimate

$$
\int_{B_{R}^{M}} d\left(\pi_{\mathbf{a}}(v), \pi_{\mathbf{a}}(\omega)\right)^{-s} d \mu(\mathbf{a}) \leqslant C(\mathbb{G}, R)
$$

for all $\omega, v \in W_{\infty, M}$ such that $v_{1} \neq \omega_{1}$. Here we used the relation (2.4) together with the effect of similarity mappings on the sub-Riemannian metric $d$.

Let us therefore fix two words $\omega, v \in W_{\infty, M}$ such that $\omega_{1} \neq v_{1}$. Without loss of generality, we can assume that $\omega_{1}=1$. By the left invariance of the sub-Riemannian metric $d$, the integrand in $(5 \cdot 12)$ is equal to

$$
d\left(\pi_{\mathbf{a}}(v), \pi_{\mathbf{a}}(\omega)\right)^{-s}=d\left(T_{\omega, v}^{1}(\mathbf{a}), 0\right)^{-s}
$$

where $T_{\omega, v}: \mathbb{G}^{M} \rightarrow \mathbb{G}^{M}$ is defined as in (4.2). Implementing the change of variables given by $T_{\omega, v}$, we estimate

$$
\begin{aligned}
\int_{B_{R}^{M}} d\left(\pi_{\mathbf{a}}(v), \pi_{\mathbf{a}}(\omega)\right)^{-s} d \mu(\mathbf{a}) & \leqslant \int_{B_{C R}^{M}} d\left(a_{1}, 0\right)^{-s} d\left(\left(T_{\omega, v}\right)_{\#} \mu\right)(\mathbf{a}) \\
& =\int_{0}^{\infty}\left(T_{\omega, v}\right)_{\#} \mu\left(\left\{\mathbf{a} \in B_{C R}^{M}: d\left(a_{1}, 0\right)^{-s}>t\right\}\right) d t \\
& =\int_{0}^{\infty}\left(T_{\omega, v}\right)_{\#} \mu\left(\left\{\mathbf{a} \in B_{C R}^{M}: d\left(a_{1}, 0\right)<t^{-1 / s}\right\}\right) d t \\
& =\int_{0}^{\infty} \mu\left(T_{\omega, v}^{-1}\left(B_{t^{-1 / s} \wedge C R} \times B_{C R}^{M-1}\right)\right) d t \\
& \leqslant C(R)+\int_{\varrho^{-1}(C R)}^{\infty} \mu\left(\Omega_{\omega, v}(\varrho(t))\right) d t
\end{aligned}
$$

where $\varrho(t)=t^{-1 / s}$. 
For each $i=1, \ldots, S$ we apply Lemma $5 \cdot 1$ to bound the integrand $\mu\left(\Omega_{\omega, v}(\varrho(t))\right)$. An application of (5.9) yields that $\int_{\varrho^{-1}(C R)}^{\infty} \mu\left(\Omega_{\omega, v}(\varrho(t))\right) d t$ is bounded above by

$$
\int_{t_{0}}^{\infty} \varrho(t)^{-i(Q M-N)-\sum_{h=1}^{i}(i-h) m_{h}+i q} d t=\int_{t_{0}}^{\infty} t^{-\frac{1}{s}\left(i q-i(Q M-N)-\sum_{h=1}^{i}(i-h) m_{h}\right)} d t,
$$

where $t_{0}:=\varrho^{-1}(C R)$. This integral converges precisely when

$$
-\frac{1}{s}\left(i q-i(Q M-N)-\sum_{h=1}^{i}(i-h) m_{h}\right)<-1
$$

or

$$
q>Q M-N+\frac{1}{i}\left(s+\sum_{h=1}^{i}(i-h) m_{h}\right) .
$$

We may choose $i$ so that the right-hand side of this inequality is equal to

$$
Q M-\left(N-\beta_{-}^{-1}(s)\right) .
$$

See (5.5). The choice of $q$ in (5.8) guarantees that (5.14) holds for this value of $i$. Then the integral in $(5 \cdot 13)$ is finite. This completes the proof of Theorem $1 \cdot 1$.

Remark $5 \cdot 1$. Corollary $1 \cdot 1$ follows from Theorem $1 \cdot 1$ by expressing $E\left(d_{\mathbf{r}}\right)$ as a countable union of sets $E(s), s<d_{\mathbf{r}}$, and using the countable stability of the Hausdorff dimension.

\section{Final comments and questions}

In this final section, we collect several comments and remarks concerning Theorem $1 \cdot 1$ and its proof, and indicate some directions for future research.

Remark 6.1. Can the estimate in Theorem $1 \cdot 1$ be improved? The appearance of the dimension comparison functions $\beta_{ \pm}$in (1.12) stems from our method of proof, which uses the Euclidean bi-Lipschitz property of the transition map $T_{\omega, v}$. The transition map cannot be expected to be bi-Lipschitz continuous for the Carnot-Carathéodory metric. In fact, the mapping $\mathbb{G} \times \mathbb{G} \rightarrow \mathbb{G},(a, b) \mapsto a * b$ is not Lipschitz continuous for the Carnot-Carathéodory metric, which is left but in general not right invariant. We do not know whether the use of dimension comparison theorems and the concomitant appearance of the functions $\beta_{ \pm}$in Theorem $1 \cdot 1$ is necessary.

Remark 6.2. Theorem $1 \cdot 1$ provides a quantitative estimate on the size of the exceptional set $E(s)$ associated with the almost sure identity

$$
\operatorname{dim}_{c c} K_{\mathbf{a}}=d_{\mathbf{r}} \quad \text { for a.e. } \mathbf{a} \in \mathbb{G}^{M} .
$$

Following the discussion in the introduction we pose two additional problems of similar type. Recall that

$$
\operatorname{dim}_{E} K_{\mathbf{a}}=\beta_{-}^{-1}\left(d_{\mathbf{r}}\right) \quad \text { for a.e. } \mathbf{a} \in \mathbb{G}^{M}
$$

We are led to pose

Problem 6.1. For $s<d_{\mathbf{r}}$, estimate the dimension of the exceptional set

$$
E^{\prime}(s)=\left\{\mathbf{a} \in \mathbb{G}^{M}: \operatorname{dim}_{E} K_{\mathbf{a}}<\beta_{-}^{-1}(s)\right\} .
$$


In fact, we may look for estimates of either the sub-Riemannian Hausdorff dimension or the Euclidean Hausdorff dimension of $E^{\prime}(s)$. Any results which we obtain for either of these problems will necessarily be related to each other through the dimension comparison theorem of [5] applied on the product Carnot group $\mathbb{G}^{M}$. We seek estimates of the form $\operatorname{dim}_{c c} E^{\prime}(s) \leqslant Q M-\varphi(s)$ or $\operatorname{dim}_{E} E^{\prime}(s) \leqslant N M-\psi(s)$. An easy computation shows that

$$
\beta_{ \pm}^{\mathbb{G}^{M}}(\alpha)=M \beta_{ \pm}^{\mathbb{G}}(\alpha / M)
$$

for $0<\alpha<N^{M}$. It follows that

$$
\operatorname{dim}_{c c} E^{\prime}(s) \leqslant Q M-\varphi(s) \Rightarrow \operatorname{dim}_{E} E^{\prime}(s) \leqslant N M-\left(\beta_{+}^{\mathbb{G}^{M}}\right)^{-1}(\varphi(s))
$$

and

$$
\operatorname{dim}_{E} E^{\prime}(s) \leqslant N M-\psi(s) \Rightarrow \operatorname{dim}_{c c} E^{\prime}(s) \leqslant Q M-\left(\beta_{-}^{\mathbb{G}^{M}}\right)(\psi(s)) .
$$

Combining (6.1) and (6.2) yields

$$
\operatorname{dim}_{c c} K_{\mathbf{a}}=\beta_{-}\left(\operatorname{dim}_{E} K_{\mathbf{a}}\right) \quad \text { for a.e. } \mathbf{a} \in \mathbb{G}^{M} ;
$$

i.e., almost every $K_{\mathbf{a}}$ is a horizontal set (in the terminology introduced in [5]). We would like to understand more precisely the prevalence of horizontal sets among sub-Riemannian self-similar fractals. We pose

Problem 6.2. For $\delta>0$ estimate the (sub-Riemannian or Euclidean) Hausdorff dimension of the exceptional set

$$
E^{\prime \prime}(\delta)=\left\{\mathbf{a} \in \mathbb{G}^{M}: \operatorname{dim}_{c c}\left(K_{\mathbf{a}}\right)>\beta_{-}\left(\operatorname{dim}_{E} K_{\mathbf{a}}\right)+\delta\right\} .
$$

Problems $6 \cdot 1$ and $6 \cdot 2$ are more difficult to tackle than Theorem $1 \cdot 1$. The reason is that these problems ask for dimension estimates in the Euclidean metric on $\mathbb{G}$, where $K_{\mathbf{a}}$ is the invariant set for a highly nonlinear IFS comprised of polynomial mappings of large degree. Clearly, any progress on either of these problems yields conclusions for the other problem, in view of Theorem $1 \cdot 1$.

Example 6.1. We conclude with an example. The setting is the simplest nonabelian Carnot group, the first Heisenberg group $\mathbb{H}^{1}$. Recall that $\mathbb{H}^{1}$ is the unique step two Carnot group of dimension three. Explicitly, $\mathbb{H}^{1}=\mathbb{R}^{3}$ with Lie algebra $\mathrm{h}^{1}=\operatorname{span}\{X, Y, T\}$, where $[X, Y]=T$. We denote by $*$ the group law in $\mathbb{H}^{1}$. Elements of $\mathbb{H}^{1}$ will be written in coordinates as $(x, y, t)$ corresponding to the basis elements in $\mathrm{h}^{1}$. For the sub-Riemannian metric on $\mathbb{H}^{1}$ we use the Korányi metric $d(p, q)=\left|p^{-1} * q\right|$ where

$$
|(x, y, t)|=\left(\left(x^{2}+y^{2}\right)^{2}+16 t^{2}\right)^{1 / 4} .
$$

The dilations $\left(\delta_{r}\right)_{r>0}$ act on $\mathbb{H}^{1}$ in the usual manner: $\delta_{r}(x, y, t)=\left(r x, r y, r^{2} t\right)$. Let $\pi_{1}$ : $\mathbb{H}^{1} \rightarrow \mathbb{R}^{2}, \pi_{1}(x, y, t)=(x, y)$ denote the projection to the first (horizontal) layer.

Let $\mathbf{f}=\left\{f_{1}, f_{2}\right\}$ be a two element IFS on $\mathbb{H}^{1}$ with contraction ratios $r_{1}, r_{2}<1 / 2$ and translation parameters $a_{1}, a_{2} \in \mathbb{H}^{1}$. The fixed point $p_{j}$ for $f_{j}$ satisfies $p_{j}=a_{j} * \delta_{r_{j}}\left(p_{j}\right)$. The similarity dimension $d_{\mathbf{r}}$ solves the equation $r_{1}^{d_{\mathbf{r}}}+r_{2}^{d_{\mathbf{r}}}=1$. In $\mathbb{H}^{1}$ the lower dimension comparison function is $\beta_{-}(\alpha)=\max \{\alpha, 2 \alpha-2\}$; thus $\beta_{-}^{-1}(s)=s$ for $s<1$. The estimate coming from Theorem $1 \cdot 1$ is

$$
\operatorname{dim}_{c c} E(s) \leqslant 2 Q-N+s=5+s,
$$


where $Q=4$ and $N=3$ are the homogeneous and topological dimensions of $\mathbb{H}^{1}$, respectively. In this example, the sub-Riemannian and Euclidean dimensions of the invariant set can be evaluated exactly for every $\mathbf{a}$ :

(i) when $p_{1}=p_{2}$ the invariant set is a point, hence has dimension zero;

(ii) when $p_{1}$ and $p_{2}$ are distinct points satisfying $\pi_{1}\left(p_{1}\right)=\pi_{1}\left(p_{2}\right)$, the invariant set $K$ is a Cantor set contained in the vertical line $\pi_{1}^{-1}\left(\pi_{1}\left(p_{j}\right)\right)$ in $\mathbb{H}^{1}$. Its sub-Riemannian dimension, $\operatorname{dim}_{c c} K$, is the similarity dimension $d_{\mathbf{r}}$, while its Euclidean dimension, $\operatorname{dim}_{E} K$, is $d_{\mathbf{r}} / 2$. In fact, $K$ is a Euclidean self-similar fractal generated by an IFS with contraction ratios $r_{1}^{2}$ and $r_{2}^{2}$;

(iii) finally, when $p_{1}$ and $p_{2}$ are distinct points for which $\pi_{1}\left(p_{1}\right) \neq \pi_{1}\left(p_{2}\right)$, the invariant set $K$ is a Cantor set satisfying $\operatorname{dim}_{c c} K=\operatorname{dim}_{E} K=d_{\mathbf{r}}$. Indeed, $\pi_{1}(K)$ is a Cantor set in $\mathbb{R}^{2}$ generated by an IFS with contraction ratios $r_{1}$ and $r_{2}$. This Euclidean IFS satisfies the open set condition, hence $\operatorname{dim}_{E} \pi_{1}(K)=d_{\mathbf{r}}$. Since id : $\left(\mathbb{H}^{1}, d\right) \rightarrow$ $\left(\mathbb{R}^{3}, d_{E}\right)$ is locally Lipschitz, we find

$$
d_{\mathbf{r}}=\operatorname{dim}_{E} \pi_{1}(K) \leqslant \operatorname{dim}_{E} K \leqslant \operatorname{dim}_{c c} K \leqslant d_{\mathbf{r}}
$$

and equality holds throughout. Note that $\pi_{1}(K)$ is contained in a one-dimensional affine subspace of $\mathbb{R}^{2}$, hence $K$ is contained in a two-dimensional vertical affine subspace of $\mathbb{H}^{1}=\mathbb{R}^{3}$.

The exceptional sets are $E(s)=\left\{\mathbf{a}=\left(a_{1}, a_{2}\right) \in \mathbb{H}^{1} \times \mathbb{H}^{1}: a_{1}=a_{2}\right\}$,

$$
E^{\prime}(s)=\left\{\mathbf{a}=\left(a_{1}, a_{2}\right) \in \mathbb{H}^{1} \times \mathbb{H}^{1}: \pi_{1}\left(a_{1}\right)=\pi_{1}\left(a_{2}\right)\right\}
$$

and

$$
E^{\prime \prime}(\delta)=\left\{\mathbf{a}=\left(a_{1}, a_{2}\right) \in \mathbb{H}^{1} \times \mathbb{H}^{1}: \pi_{1}\left(a_{1}\right)=\pi_{1}\left(a_{2}\right), a_{1} \neq a_{2}\right\}
$$

for $0<s<d_{\mathbf{r}}$ and $\delta>0$ sufficiently small. Then $\operatorname{dim}_{c c} E(s)=4$ and

$$
\left(\operatorname{dim}_{E} E^{\prime}(s), \operatorname{dim}_{c c} E^{\prime}(s)\right)=\left(\operatorname{dim}_{E} E^{\prime \prime}(\delta), \operatorname{dim}_{c c} E^{\prime \prime}(\delta)\right)=(4,6) .
$$

Note that the actual value of $\operatorname{dim}_{c c} E(s)$ is smaller than the prediction of Theorem 1.1 by a definite amount, regardless of the value of $s$.

Remark 6.3. As indicated above, the invariant set for any two element IFS in $\mathbb{H}^{1}$ lies in a vertical affine plane, i.e., a coset of a vertical two-dimensional homogeneous subgroup $P$. By homogeneous we mean that $P$ is closed under the action of the dilations $\left(\delta_{r}\right)_{r>0}$. Any such subgroup is spanned by a horizontal element $V \in \operatorname{span}\{X, Y\}$ together with the nonhorizontal vector $T$. Observe that $P$ can be identified with $\mathbb{R}^{2}$ via coordinates $(v, t)$, and that the restriction of the Korányi metric to $P$ is bi-Lipschitz equivalent to the parabolic (heat) metric $d_{\text {heat }}\left(\left(v_{1}, t_{2}\right),\left(v_{2}, t_{2}\right)\right)=\left|v_{1}-v_{2}\right|+\sqrt{\left|t_{1}-t_{2}\right|}$ on $\mathbb{R}^{2}$. Any two such cosets are equivalent via an isometry of $\mathbb{H}^{1}$. Finally, any two points in $\mathbb{H}^{1}$ are contained in such a coset. In view of these remarks, we consider the results of [5] and this paper in the context of such homogeneous subgroups, equipped with their natural dilation structures and left invariant homogeneous metrics. Fix a particular subgroup $P$ of this type, e.g., $P=\operatorname{span}\{X, T\}$. Observe that the relevant dimensions of $P$ are $N=2$ (topological dimension) and $Q=3$ (homogeneous dimension). Then $E(s)=\left\{\mathbf{a} \in P \times P: a_{1}=a_{2}\right\}$ has $\operatorname{dim}_{c c} E(s)=Q=3$, while Theorem 1.1 predicts $\operatorname{dim}_{c c} E(s) \leqslant 2 Q-N+s=4+s$. Again we observe the discrepancy between the prediction and the exact value, which ultimately stems from the method of proof via the Euclidean bi-Lipschitz property of the transition map and the use of dimension comparison theorems. 
Acknowledgements. Research for this paper was completed while the third and fourth authors were visitors in the Mathematisches Institut, Universität Bern. The authors would like to thank the institute for its gracious hospitality.

\section{REFERENCES}

[1] Z. M. BAlogh, R. Hoefer-Isenegger and J. T. Tyson. Lifts of Lipschitz maps and horizontal fractals in the Heisenberg group. Ergodic Theory Dynam. Systems, 26, 3 (2006), 621-651.

[2] Z. M. BALOGH and H. ROHNER. Self-similar sets in doubling spaces. Illinois J. Math (2007), 12751297.

[3] Z. M. BALOGH and J. T. TYSON. Hausdorff dimensions of self-similar and self-affine fractals in the Heisenberg group. Proc. London Math. Soc. (3) 91, 1 (2005), 153-183.

[4] Z. M. BALOGH, J. T. TYSON and B. WARHURST. Gromov's dimension comparison problem on Carnot groups. C. R. Math. Acad. Sci. Paris 346, 3-4 (2008), 135-138.

[5] Z. M. BAlogh, J. T. TySON and B. WARhurst. Sub-Riemannian vs. Euclidean dimension comparison and fractal geometry on Carnot groups. Adv. Math. 220, 2 (2009), 560-619.

[6] A. Bonfiglioli, E. LANConelli and F. Uguzzoni. Stratified Lie groups and potential theory for their sub-Laplacians. Springer Monographs in Math. (Springer, Berlin, 2007).

[7] L. J. CORWIN and F. P. GREENLEAF. Representations of nilpotent Lie groups and their applications. Cambridge Studies in Adv. Math. Part I, vol. 18 (Cambridge University Press, 1990).

[8] K. FAlConer and J. MiaO. Exceptional sets for self-affine fractals. Math. Proc. Camb. Phil. Soc. 145, 3 (2008), 669-684.

[9] K. J. FALCONER. The geometry of fractal sets, Cambridge Tracts in Mathematics. (Cambridge University Press, 1986) vol. 85.

[10] K. J. FAlconer. The Hausdorff dimension of self-affine fractals. Math. Proc. Camb. Phil. Soc. 103 (1988), 339-350.

[11] K. J. FALCONER. Fractal geometry. Mathematical Foundations and Applications. (John Wiley and Sons Ltd., 1990).

[12] K. J. FAlCONER. The dimension of self-affine fractals. II. Math. Proc. Camb. Phil. Soc. 111 (1992), 169-179.

[13] G. B. Folland and E. M. Stein. Hardy Spaces on Homogeneous Groups. (Princeton University Press, 1982).

[14] M. Gromov Carnot-Carathéodory spaces seen from within. In Sub-Riemannian Geomet. Progr. Math. 144 (1996), pp. 79-323.

[15] J. E. Hutchinson. Fractals and self-similarity. Indiana Univ. Math. J. 30, 5 (1981), 713-747.

[16] J. Kigami. Analysis on fractals. Cambridge Tracts in Mathematics. Cambridge University Press, 2001 vol. 143.

[17] P. Mattila. Geometry of sets and measures in Euclidean spaces, vol. 44 of Cambridge Studies in Advanced Mathematics. (Cambridge University Press, 1995). Fractals and rectifiability.

[18] P. MatTila Measures with unique tangent measures in metric groups. Math. Scand. 97, 2 (2005), 298-308.

[19] P. Mattila, R. Serapioni and F. Serra Cassano. Characterizations of intrinsic rectifiability in Heisenberg groups. Ann. Scuola Norm. Sup. Pisa Cl. Sci. (4). to appear.

[20] R. MONTGOMERY A tour of subriemannian geometries, their geodesics and applications. No. 91 in Mathematical Surveys and Monographs (Amer. Math. Soc., 2002).

[21] Y. PERES and W. Schlag. Smoothness of projections, Bernoulli convolutions, and the dimension of exceptions. Duke Math. J. 102, 2 (2000), 193-251.

[22] B. SolOMYAK. Measure and dimension for some fractal families. Math. Proc. Camb. Phil. Soc. 124, 3 (1998), 531-546.

[23] R. S. STRICHARTZ. Self-similarity on nilpotent Lie groups. In Geometric analysis (Philadelphia, PA, 1991), Contemp. Math. 140 (1992), pp. 123-157. 